\title{
Nouvelles données historiques sur les premiers dinosaures trouvés en France
}

\author{
Arnaud Brignon* \\ 5 villa Jeanne d'Arc, 92340 Bourg-la-Reine, France
}

Reçu le 22 mai 2017 / Accepté le 29 janvier 2018

\begin{abstract}
Résumé - Cet article passe en revue les premières découvertes de dinosaures faites en France avant que le terme Dinosauria n'ait été introduit par Richard Owen en 1842. En mettant à part les premières découvertes du théropode Streptospondylus altdorfensis faites au XVIII ${ }^{\mathrm{e}}$ siècle par Charles Bacheley dans le Jurassique des Vaches Noires, les principaux résultats démontrés ici sont les suivants (par ordre chronologique) : 1) les premiers restes de dinosaures (dents de théropodes) du Calcaire de Caen (Bathonien) furent collectés par Arcisse de Caumont dans les carrières de Quilly en, ou avant, avril 1826 et la première mention de cette découverte fut publiée en 1827 ; 2) les restes de théropode que William Buckland avait observés en octobre 1826 dans le Cabinet d'Histoire naturelle de Besançon furent signalés dans une publication dès 1830 ; 3) la première mention à un dinosaure du Jurassique supérieur du Boulonnais fut longtemps considérée avoir été celle faite par Constant Prévost en 1839. Des dents d'un théropode et d'un dinosaure herbivore furent, semble-t-il, découvertes dans ces formations par Eugène Robert en automne 1833. Une autre dent de théropode, celle-ci identifiable de manière incontestable, fut collectée en, ou avant, juillet 1835 par Bruno Marmin comme en atteste une lettre inédite à Jules Desnoyers; 4) Le genre Poekilopleuron EudesDeslongchamps, 1836 et son espèce type $P$. bucklandii Eudes-Deslongchamps, 1836, du Calcaire de Caen (Bathonien moyen), ont été créés de manière valide en 1836;5) l'humérus de sauropode découvert dans le Crétacé supérieur de la plaine de Lisle près de Périgueux et figuré par Paul Gervais en 1852 fut donné au Muséum d'Histoire naturelle à Paris par un certain Simon Bornet en 1841;6) l'humérus de sauropode du grès vert (Albien) de Bédoin près du mont Ventoux, décrit par Gervais en 1852 sous le nom d'Aepisaurus elephantinus, fut découvert par Prosper Renaux en 1841. Ce dernier effectua des dessins du spécimen à partir desquels une lithographie restée inédite fut réalisée. Renaux est ainsi le premier à avoir découvert et étudié un dinosaure, authentifié de manière incontestable, dans le Crétacé provençal. Les affinités de cet os avec les genres Megalosaurus et Iguanodon furent reconnues par Henri Marie Ducrotay de Blainville dès 1842.
\end{abstract}

Mots clés : histoire de la paléontologie / Dinosauria / Theropoda / Sauropoda / Jurassique / Crétacé

Abstract - New historical data on the first dinosaurs found in France. This article reviews the earliest dinosaur discoveries in France before the term Dinosauria was coined by Richard Owen in 1842. Leaving aside the first discoveries of the theropod Streptospondylus altdorfensis made during the eighteenth century by Charles Bacheley in the Jurassic of the Vaches Noires (Normandy), the main results shown in the present paper are the following (in chronological order): 1) the first dinosaur remains (theropod teeth) from the Calcaire de Caen (Bathonian) were collected by Arcisse de Caumont in the Quilly quarries in or before April 1826. The first mention of this discovery was published in 1827 and not in 1828 as previously thought; 2) the theropod remains observed by William Buckland in October 1826 in the Cabinet of Natural History of Besançon were reported in a publication in 1830; 3) The first mention of a dinosaur from the Upper Jurassic of Boulogne-sur-Mer was long considered to be the one made by Constant Prévost in 1839. However, a theropod tooth and another one from a supposed herbivorous dinosaur were apparently discovered in these formations by Eugène Robert as early as autumn 1833. Another theropod tooth, identifiable beyond doubt, was collected in or before July 1835 by Bruno Marmin as evidenced by an unrecorded letter to Jules Desnoyers; 4) the genus Poekilopleuron Eudes-Deslongchamps, 1836 and its type species P. bucklandii Eudes-Deslongchamps, 1836, from the Calcaire de Caen (Middle Bathonian), were validly introduced in $1836 ; 5)$ the sauropod humerus from the Late Cretaceous of the plain of Lisle near Périgueux (Dordogne),

*Auteur correspondant : arnaud.brignon@yahoo.com 
figured by Paul Gervais in 1852, was offered to the Muséum d'Histoire naturelle, Paris by a certain Simon Bornet in 1841; 6) the sauropod humerus from the Greensand (Albian) of Bédoin, near Mont Ventoux, described by Paul Gervais in 1852 under the name Aepisaurus elephantinus was discovered by Prosper Renaux in 1841. The latter made some drawings of the bone from which an unpublished lithograph was realized. Renaux was the first to discover and study a verified dinosaur bone from the Cretaceous of Provence. The affinity of this bone with the genera Megalosaurus or Iguanodon was recognized by Henri Marie Ducrotay de Blainville as early as 1842.

Keywords: history of palaeontology / Dinosauria / Theropoda / Sauropoda / Jurassic / Cretaceous

\section{Introduction}

L'histoire des premières découvertes de restes de dinosaures, avant que le terme Dinosauria ne soit introduit par Richard Owen (1804-1892) en 1842 (Owen, 1842), a fait l'objet d'une abondante littérature (Delair et Sarjeant, 1975, 2002 ; Dean, 1999; Moody et al., 2010 ; Spalding et Sarjeant, 2012; Sues, 2012). Les informations disponibles sur ces découvertes faites en France ont été principalement compilées dans les années 1980 et 1990 (Buffetaut, 1983, 1995 ; Taquet, 1984, 1994; Buffetaut et al., 1991, 1993, 1994; Taquet et Contini, 1997) mais n'ont pas été révisées depuis.

Cet article vise à apporter des renseignements inédits ou à préciser certaines données sur les premières découvertes de dinosaures faites en France jusqu'au tout début des années 1840. Ces informations s'appuient sur une révision de la littérature scientifique du XIX ${ }^{\mathrm{e}}$ siècle, des éléments biographiques trouvés dans différentes archives départementales et des documents découverts dans les fonds patrimoniaux conservés à la Bibliothèque centrale du Muséum nationale d'Histoire naturelle (Paris) et à la Bibliothèque nationale de France.

\section{Premières découvertes dans la série callovo-oxfordienne des Vaches Noires}

L'abbé Charles Bacheley (1716-1795), dont la biographie a été récemment dévoilée (Brignon, 2016a), est le premier naturaliste à avoir collecté des restes de dinosaures en France de manière incontestable (Brignon, 2016b). Ces ossements, attribués plus tard au théropode Strepospondylus altfordensis Meyer, 1832, furent trouvés dans les marnes callovooxfordiennes (Callovien supérieur/Oxfordien inférieur) au pied des falaises des Vaches-Noires entre Villers-sur-Mer et Dives-sur-Mer (Bacheley, 1778). Charles Bacheley est par conséquent le premier (Brignon, 2016b), avant William Buckland (1784-1856) et Gideon Mantell (1790-1852) en Angleterre, à s'être procuré des restes de dinosaures présentant suffisamment de caractères diagnostiques pour être utilisés comme matériel-type d'un genre et d'une espèce valides (Allain, 2001; Carrano et al., 2012).

Un autre ecclésiastique français, Jacques-François Dicquemare (1733-1789), avait peut-être lui aussi trouvé des restes de dinosaures dans le Jurassique des Vaches Noires (Dicquemare, 1776), mais cette hypothèse n'est fondée que sur la description d'un supposé fémur de grande dimension (Taquet, 1984, 1994, p. 272). Un grand nombre de spécimens collectés par l'abbé Bacheley ont, quant à eux, bel et bien été figurés et décrits en détail par Georges Cuvier (1808), et plusieurs d'entre eux correspondent sans ambiguïté à des restes de dinosaures. Ces pièces sont encore conservées dans les collections du MNHN et comprennent notamment des vertèbres opisthocoeles, dont Cuvier avait parfaitement noté la singularité par rapport à celles des crocodiles actuels (Allain, 2001). Cuvier peut de ce fait être considéré comme le premier à avoir donné une description scientifique de restes de dinosaures, contrairement à l'opinion communément admise (Delair et Sarjeant, 2002), qui attribue cette primeur à William Buckland (1824). À une époque où la notion de dinosaures n'avait pas encore été introduite, le savant parisien considérait les restes du théropode de la collection Bacheley comme étant ceux d'une «espèce inconnue de crocodile» alors que le révérend britannique estimait que son Megalosaurus était un «lézard géant». On ne saurait hiérarchiser ces travaux sur cette seule base même s'il est vrai que Buckland, aidé par Conybeare, a introduit le premier genre de dinosaure considéré comme valide (Benson et al., 2008).

\section{Les dinosaures du Bathonien du Calvados}

Après les premières découvertes de dinosaures faites aux Vaches Noires, c'est ensuite à Alcide de Caumont que l'on doit le signalement de dents de théropodes dans le Calcaire de Caen (Bathonien moyen) (Buffetaut et al., 1993). La partie sommitale de cette formation correspond aux couches de pierre de taille, ou Pierre de Caen, qui fut exploitée de manière intensive jusqu'au début du $\mathrm{xx}^{\mathrm{e}}$ siècle (Dugué et al., 2010). Cette pierre était extraite principalement à ciel ouvert jusqu'au $\mathrm{XVII}^{\mathrm{e}}$ siècle, mais l'épuisement des gisements conduisit à son exploitation dans des carrières souterraines, notamment près du village d'Allemagne (renommé Fleury-sur-Orne en 1917), au hameau de La Maladrerie (devenu depuis un quartier de Caen) et enfin à Quilly, entre Bretteville-sur-Laize et les Cintheaux. La Pierre de Caen qui débute par le banc royal d'un mètre d'épaisseur environ a fourni de nombreux restes de vertébrés (thalattosuchiens, dinosauriens, sauroptérygiens, ichthyosauriens, téléostéens et sélaciens). La découverte de crocodylomorphes marins (Thalattosuchia) presque complets dans cette formation eut un grand retentissement au début du $\mathrm{XIX}^{\mathrm{e}}$ siècle. Le squelette d'un Teleosaurus cadomensis découvert en 1817 près du village d'Allemagne ainsi que l'empreinte et quelques restes d'un Steneosaurus megistorhynchus trouvé à Quilly en 1822 constituaient les spécimens les plus remarquables (Brignon, 2014). La découverte des premiers restes de théropodes dans la Pierre de Caen par Alcide de Caumont est généralement datée de 1828, date de la publication de son Essai sur la topographie géognostique du département du Calvados publié dans les Mémoires de la Société Linnéenne de Normandie (Buffetaut et al., 1991, 1993 ; Buffetaut 1995, p. 58 ; 2011, p. 24). Alcide de Caumont 
(1828, p. 207) y signale «des débris de Megalosaurus». Sur une des planches qui accompagnent l'atlas de ce journal pour l'année 1828, une dent de théropode est également figurée sans indication (Caumont, 1828, pl. 8, «dent de Megalosaurus » et «coupe transversale de la dent»). Le lien entre le travail d'Arcisse de Caumont et cette figure fut confirmé plus tard, en 1833 (Anonyme, 1833, p. 40), puis en 1837 par JacquesAmand Eudes-Deslongchamps $(1837$ a, p. $7 ; 1838$, p. 39) à qui de Caumont avait donné la fameuse dent. Cette dernière fut ensuite déposée dans les collections de la Faculté des Sciences de Caen (Gervais, 1848-1852, vol. 1, p. 264) où elle fut détruite lors des bombardements de 1944 (Bigot, 1945, p. 29).

Il est en revanche peu connu que cette découverte fut mentionnée dans le rapport des travaux de la Société Linnéenne de Normandie, lu par de Caumont durant la séance publique du 24 mai 1826. Ce rapport, publié en 1827, relate que de Caumont (1827, p. xxix) avait «présenté des dents de Mégalosaurus découvertes dernièrement dans les carrières de Quilly (Calvados)». Le fonds Cuvier de la Bibliothèque centrale du MNHN conserve par ailleurs une lettre d'Arcisse de Caumont à Cuvier qui démontre que le géologue normand avait signalé dès le mois d'avril 1826 la découverte de dents de Megalosaurus dans le Calcaire de Caen (Fig. 1). Cette lettre n'est pas datée mais est associée à un document imprimé daté du 8 avril 1826 invitant les membres correspondants de la Société Linnéenne de Normandie, dont Cuvier faisait partie, à soumettre des articles dans le journal édité par cette société savante. La lettre est adressée à «Monsieur le Baron Cuvier, Secrétaire perpétuel de l'Académie des Sciences, 39 rue de Seine, Paris ». On peut y lire : «Je prends la liberté de vous prévenir, Monsieur, que je viens de trouver des dents de Mégalausaurus [sic] dans le calcaire de Caen. J'ai réuni de ce calcaire un grand nombre d'autres productions qui presque toutes sont identiques avec celle de Stonesfield en Angleterre, et je me propose de publier une note sur cette analogie. Je me trouve heureux de pouvoir vous annoncer ces faits géologiques qui peut-être vous présenteront quelque intérêt et $\mathrm{j}$ 'ai l'honneur d'être avec un profond respect, Monsieur, votre très humble et très obéissant serviteur, de Caumont». Arcisse de Caumont avait fort justement observé la similarité de la faune fossile de "l'oolithe de Stonesfield» (Taynton Limestone Formation, Bathonien moyen) (Boneham et Wyatt, 1993; Allain, 2002) avec celle du Calcaire de Caen.

Une autre découverte, encore plus spectaculaire, faite dans la Pierre de Caen extraite des anciennes carrières souterraines de La Maladrerie, survint en juillet 1835. Jacques-Amand Eudes-Deslongchamps parvint à rassembler le squelette partiel d'un dinosaure auquel il donna le nom de Poekilopleuron bucklandii. Il est inutile de revenir sur les circonstances rocambolesques de cette découverte qui sont bien documentées par ailleurs (Eudes-Deslongchamps, 1837a, 1838; Taquet, 1994 , p. 283-287 ; Buffetaut, 1995, p. 58-60 ; 2001, p. 34-36; Allain, 2002). Il est en revanche intéressant de revenir un instant sur la biographie mal connue de Bourienne, docteur en médecine, qui observa les premiers ossements du dinosaure et attira l'attention de son collègue Eudes-Deslongchamps sur cette importante découverte. Alexandre Frédéric Bourrienne (1797-1871) naquit à Moulins-sur-Orne près d'Argentan (Orne) le 6 floréal an V (25 avril 1797) (Archives départementales de l'Orne, Moulins-sur-Orne, registre d'état civil, 1793an V, 3E2-298/3). Fils de Philippe Pierre Bourrienne, cultivateur, et de Charlotte David, il se maria à Caen le 10 novembre 1823 avec Justine Levesque (ADC, Caen, Mariage, 1823, 2 MI-EC 1620). Il faisait partie des premiers membres résidents lors de la fondation, en 1823, de la Société Linnéenne de Normandie dont il fut le président en 1830 et le vice-président en 1850. Bourienne s'éteignit à Caen le 25 février 1871 à l'âge de 73 ans (ADC, Caen, décès, 1871, 2 MI-EC 15). Son fils Alexandre Bourienne, né le 6 octobre 1829 (ADC, Caen, Naissance, 1829, 2 MI-EC 1628), fut également élu membre de la Société Linnéenne de Normandie en 1854

Il existait encore jusqu'à présent une certaine confusion au sujet de la date de publication du «Mémoire sur le Poekilopleuron bucklandii» d'Eudes-Deslonchamps qui est indiquée comme étant tantôt l'année 1837 (Taquet, 1994), tantôt 1838, bien qu'un certain consensus semblait émerger pour cette dernière (Buffetaut, 1995, 2011; Allain et Chure, 2002; Rothschild et Tanke, 2005). Le travail d'EudesDeslonchamps était destiné aux Mémoires de la Société linnéenne de Normandie et parut en effet en 1838 dans le sixième volume de ce journal (Eudes-Deslongchamps, 1838). Cependant un tiré à part, comportant le même texte et les mêmes planches, mais avec sa propre page de titre sur lequel est explicitement indiqué «extrait du sixième volume des Mémoires de la Société Linnéenne», fut imprimé et distribué de manière anticipée en 1837 (Eudes-Deslongchamps, 1837a). Dans un article présenté durant la séance de l'Académie des Sciences du 14 août 1837, Etienne Geoffroy Saint-Hilaire (1837, p. 187; Anonyme, 1837a) signalait que l'ouvrage d'Eudes-Deslongchamps, une «brochure in $-4^{\circ}$, 112 pages avec planches », lui avait été envoyé, ainsi qu'à d'autres membres de l'académie, durant «l'avant-dernière séance», c'est-à-dire le 31 juillet 1837 (Brignon, 2013, p. 179). Le mémoire d'Eudes-Deslongchamps se retrouve ainsi indiqué dans plusieurs listes bibliographiques des ouvrages parus en 1837 et était disponible à l'achat chez les éditeurs Hardel, à Caen, et Dérache, à Paris (Arnoult, 1837 ; Pillet, 1837, p. 428429; Brockhaus, 1837; Leonhard et Bronn, 1837b; EudesDeslongchamps, 1837b). D'après l'article $21.8 \mathrm{du}$ Code International de Nomenclature Zoologique (ou «le Code» dans la suite) (ICZN, 1999) relatif à la distribution anticipée de tirés à part, l'année de publication des actes nomenclaturaux contenus dans le mémoire d'Eudes-Deslongchamps est sans ambigüité 1837 , et non 1838 .

Ce point étant traité, il n'en reste pas moins que le nom Poekilopleuron bucklandii fut introduit de manière valide par Eude-Deslongchamps dès 1836. Durant la séance publique de la Société linnéenne de Normandie qui se tenait à Vire le 24 mai 1836, le paléontologue normand lut plusieurs extraits de son mémoire sur le «très-grand animal fossile découvert», en 1835, «dans les carrières de la Maladrerie», dont la rédaction était déjà très avancée. Il présenta également 7 planches, dont plusieurs doubles, exécutées à l'encre de Chine, représentant les os du spécimen. Les extraits du mémoire d'Eudes-Deslongchamps furent publiés en 1836 dans les procès-verbaux des séances de cette société savante (Eudes-Deslongchamps, 1836a). Ces mêmes extraits, avec un texte légèrement modifié, parurent également le 21 septembre 1836 dans $L$ 'Institut, « le journal général des sociétés et travaux scientifiques de la France et de l'étranger » (Eudes-Deslongchamps, 1836b). Le travail d'Eudes-Deslongchamps, imprimé 


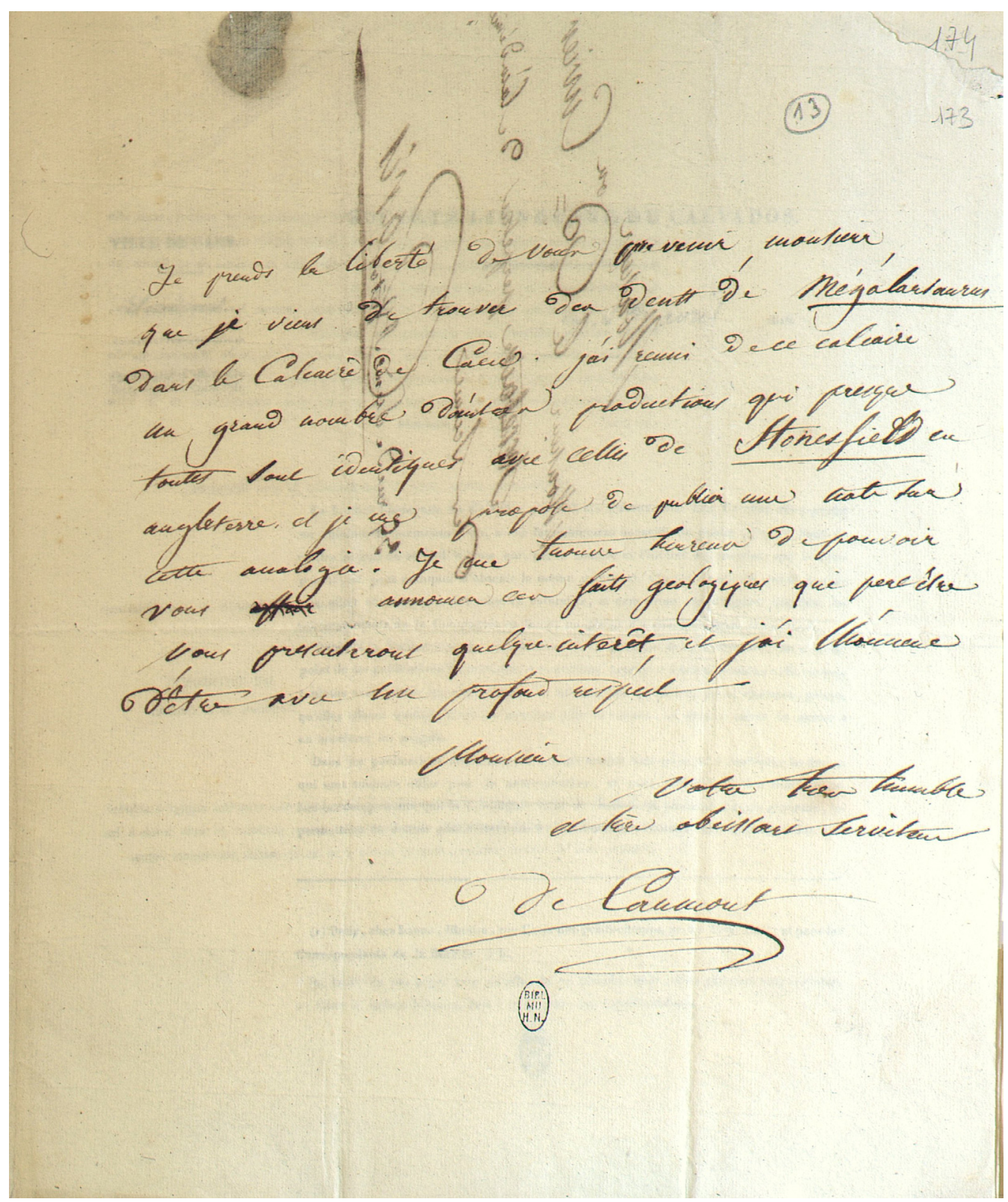

Fig. 1. Lettre inédite d'Arcisse de Caumont à Georges Cuvier (BCM Ms 633/173) annonçant, en avril 1826, la découverte de dents de «Megalausaurus [sic]» (Theropoda indéterminé) dans le Calcaire de Caen (Bathonien moyen). (C) Muséum national d'Histoire naturelle (Paris)-Direction des bibliothèques et de la documentation.

Fig. 1. Unpublished letter by Arcisse de Caumont to Georges Cuvier (BCM Ms 633/173) dated April 1826. De Caumont announces the discovery of «Megalausaurus [sic]» teeth (Theropoda indeterminate) in the Calcaire de Caen (Middle Bathonian). (C) Muséum national d'Histoire naturelle (Paris) - Direction des bibliothèques et de la documentation.

dans ces deux revues, satisfait les conditions requises par l'article 8 du Code International de Nomenclature Zoologique (ICZN, 1999), sans tomber dans les restrictions énoncées dans l'article 9, et est donc disponible au regard du Code. Le nom Poekilopleuron bucklandii y apparaît pour la première fois accompagné d'une description. D'après l'article 12.1 du Code, ce nom est par conséquent introduit de manière valide et l'année 1836 doit être retenue comme l'année de création du genre Poekilopleuron et de son espèce type par monotypie $P$. bucklandii. Le procès-verbal de la présentation d'EudesDeslongchamps, publié dans la revue «Séance Publique de la
Société Linnéenne de Normandie» (Eudes-Deslongchamps, 1836a) et dans le journal «L'Institut » (Eudes-Deslongchamps, 1836b), est rédigé dans les deux cas à la troisième personne, ce qui laisserait supposer qu'il fut retranscrit par un secrétaire dont l'identité n'est pas clairement identifié. D'après l'article 50.2 du Code (ICZN, 1999), «si le nom d'un taxon a été rendu disponible par publication dans les procès-verbaux ou comptes rendus d'une réunion, c'est le responsable du nom qui en est l'auteur, et non le secrétaire ou autre rapporteur de la réunion ». Jacques-Amand Eudes-Deslongchamps est donc bien l'auteur du nom Poekilopleuron bucklandii au regard du Code. Nous 
retiendrons la référence (Eudes-Deslongchamps, 1836a) comme la publication originale dans laquelle ce nom est rendu disponible, la référence (Eudes-Deslongchamps, 1836b) apparaissant comme une réédition.

Le nom Poekilopleuron bucklandii Eudes-Deslongchamps, 1836a circulait donc dans la littérature dès 1836 et a fortiori en 1837, avec parfois des amendements injustifiés sur l'orthographe de Poekilopleuron en «Poecilopleuron» et de bucklandii en «bucklandi» (Widenmann, 1837; Leonhard et Bronn, 1837a; Bronn, 1837, p. 522; Anonyme, 1837b; Wiegmann, 1837 , p. 230). Après la destruction des collections paléontologiques de Caen en 1944, les éléments du squelette partiel du Poekilopleuron bucklandii décrits par EudesDeslongchamps furent perdus. Seuls subsistent aujourd'hui des moulages en plâtres de quelques-unes de ces pièces préservés notamment au MNHN et au Yale Peabody Museum of Natural History (Bigot, 1945; Allain et Chure, 2002).

Une autre découverte, très peu connue, fut rapportée par Frédéric de La Fresnaye (1783-1861), de son nom complet Noël Frédéric Armand André, $3^{\mathrm{e}}$ baron de La Fresnaye (ou de Lafresnaye), ornithologue distingué et grand collectionneur d'oiseaux naturalisés (Farber, 1982, p. 64 ; BCM, Ms1987/646, lettre du baron de La Fresnaye à Georges Cuvier, $1^{\mathrm{er}}$ mars 1830). Durant la séance du 22 juillet 1833 du Congrès scientifique de France qui se tint à Caen, il présenta une portion d'os cylindrique de grande dimension tronquée aux deux extrémités, recueillie dans un banc calcaire à Épaney (et non Epone comme il est indiqué par erreur dans le compte rendu de la séance) (Anonyme, 1833, p. 40 ; Boué, 1834, p. 447). Dans cette localité, située à 8 kilomètres environ au nord de Falaise, non loin d'Aubigny et de Saint-Pierre-Canivet, étaient exploités à l'époque le Calcaire de Caen, le Calcaire de Rouvres et le Calcaire de Bon-Mesnil, trois formations rapportées au Bathonien moyen (Gigot et al., 1999). L'auteur anonyme du compte rendu, probablement Jacques-Amand Eudes-Deslongchamps en tant que secrétaire de la section d'histoire naturelle, affirma que cette portion d'os était un morceau de fémur. Il supposa qu'il devait appartenir, par sa taille, au genre Megalosaurus. La découverte du baron de La Fresnaye fut également présentée lors de la séance publique de la Société linnéenne de Normandie à Falaise le 5 juin 1834 (Caumont, 1838, p. 4). En l'absence d'illustration, le rattachement de ce spécimen à un dinosaure reste cependant hypothétique.

Revenons enfin sur un spécimen que Georges Cuvier (1824, p. 354-355, pl. 22, fig. 4) avait figuré et décrit comme l'«operculaire [splénial] du côté gauche de la mâchoire inférieure» d'un «animal gigantesque» trouvé dans «les carrières d'oolithes de Caen ». Cuvier supposa que cet élément devait appartenir à la mâchoire d'un mégalosaure. JacquesAmand Eudes-Deslongchamps (1837a, p. 93; 1838, p. 125) précisa que cette pièce provenait du Calcaire de Caen des carrières de Quilly et qu'elle avait été donnée à Cuvier par Jean-Vincent-Félix Lamouroux (1779-1825), professeur adjoint d'histoire naturelle à l'Académie de Caen. D'après les informations manuscrites laissées par Lamouroux sur les reptiles fossiles découverts dans la Pierre de Caen, le spécimen dont il est question ici fut découvert en 1821 (Brignon, 2014, p. 389, 395, spécimen \#9). Dans une note rédigée à la troisième personne, Lamouroux décrivait ainsi les circonstances de cette trouvaille (BCM Ms629/357-358) : «les ouvriers firent voir à
Mr. Lamouroux dans un banc bien inférieur, des débris saillant d'un os fossile qui avaient appartenu, disaient-ils, à un animal dont les débris avaient presque rempli un tombereau et qui occupaient un espace de plus de 20 pieds en tout sens. $\mathrm{Mr}$. Lamouroux le fit détacher avec beaucoup de peine de la roche où il était renfermé et le fit transporter dans son cabinet, où il existe encore. Il croit que c'est un fragment de mâchoire d'un très grand crocodile». Ce spécimen est répertorié dans le «Catalogue des ossements fossiles de vertébrés placés dans les galeries de géologie et minéralogie » $(1861$, p. 1405, numéro MNHN.F.AC9570) de l'ancienne collection d'anatomie comparée du Muséum d'Histoire naturelle. Il y est identifié comme appartenant au théropode Poekilopleuron bucklandii. Cette pièce est toujours conservée dans les collections de paléontologie du MNHN et correspond à un fragment de mandibule d'ichtyosaure (Ophthalmosauridae indéterminé).

\section{William Buckland et le «Mégalosaure » de Franche-Comté}

En octobre 1826, William Buckland visita le Cabinet d'Histoire naturelle de Besançon (Taquet et Contini, 1997). Buckland (1836, vol.1, p. 234) reconnut un fragment de mâchoire contenant des dents et quelques autres os qu'il identifia comme étant des restes de Megalosaurus, un genre qu'il venait de décrire deux années auparavant (Buckland, 1824). Dans les années 1990, quelques restes de théropodes furent retrouvés dans les collections paléontologiques de l'Université de Besançon, héritières des collections de l'ancien musée d'histoire naturelle de cette ville. Ce matériel comprend des vertèbres sacrées soudées (Buffetaut et al., 1994), trois métatarsiens, trois vertèbres dorsales et des morceaux de côtes (Taquet et Contini, 1997). Ces éléments proviennent, d'après les étiquettes qui les accompagnent, du Puits de Brême, sur l'actuelle commune de Scey-Maisières (Doubs), et ont été trouvés dans un niveau d'oolithes ferrugineuses d'âge Callovien. Il est fort probable que ces restes soient bien ceux que Buckland avait observés en 1826 même s'il n'existe à ce jour aucune preuve irréfutable en l'absence d'illustrations ou de documents anciens venant établir ce rapprochement. La description succincte du Cabinet d'Histoire naturelle de Besançon établit en 1828 par Frédéric Gevril (né à Locle en Suisse vers 1773-mort à Besançon le $1^{\text {er }}$ août 1856), conservateur de ce musée entre 1820 et 1844, n'apporte malheureusement pas de renseignements sur ce point (Gevril, 1828; Guenard, 1844, p. 246; Magnin, 1910, p. 471).

Il est généralement fait référence à l'édition originale de l'ouvrage Geology and Mineralogy considered with reference to natural theology, publiée en 1836, pour citer la première allusion aux restes de «Megalosaurus » que Buckland avait observés dans le Cabinet d'Histoire naturelle de Besançon en 1826 (Buffetaut et al., 1994; Buffetaut, 1995, p. 68-69; Taquet et Contini, 1997). Il est en revanche passé inaperçu que Buckland avait parlé bien avant de cette découverte au géologue britannique Henry Thomas De la Beche (1796-1855) avec qui il entretenait une correspondance suivie (Sharpe et McCartney, 1998). De la Beche mentionna ainsi la présence de ces restes dès septembre 1830, dans un article sur les fossiles des formations oolitiques d'Angleterre, du Bassin de Paris et du sud de la France, dans lequel on peut lire: «Dr. Buckland 
informs me that in the year 1826 he recognised many bones of the Megalosaurus in the Museum of Besançon from the oolite of that neighbourhood» (De la Beche, 1830a, p. 210;1830b, p. 66). L'allusion au «Megalosaurus » de Besançon fut ensuite reprise dans son manuel de géologie dont la première édition parut en 1831 (De la Beche, 1831, p. 367).

\section{Les premiers restes de dinosaures trouvés dans le Kimméridgien et le Tithonien}

Il convient de débuter ce chapitre en mentionnant une découverte faite par Charles Bertrand-Geslin (1796-1863), géologue nantais, ancien élève d'Alexandre Brongniart. Durant la séance de la Société géologique de France du 3 juin 1833, Bertrand-Geslin présenta à l'assemblée «une vertèbre de Megalosaurus qu'il a trouvé en 1831 dans l'oolithe moyenne du canal de Belle-Croix près de La Rochelle, à plus de 30 pieds $[\sim 10 \mathrm{~m}]$ de profondeur dans cette formation $»$ (Bertrand-Geslin, 1833 ; Boué, 1834, p. 447). Près du village de Belle-Croix, sur la commune de Dompierre-sur-Mer, le canal de Marans à La Rochelle traverse des calcaires du Kimméridgien inférieur (Hantzpergue et al., 1988) dans lesquels de nombreux fossiles furent collectés lors de son creusement (Cressac et Manès, 1830, p. 236). En l'absence d'une illustration et d'une description détaillée, l'attribution de cette vertèbre à un dinosaure reste cependant hypothétique. Bertrand-Geslin légua sa bibliothèque scientifique et ses collections minéralogiques et paléontologiques au Muséum d'Histoire naturelle de Nantes. En juillet 1831, dans un de ses carnets manuscrits intitulé « Voyage de Nantes à la Rochelle, Saintes, Bordeaux, Angoulême, Poitiers, Niort, Fontenay et retour à Nantes, an $1831 »$, Bertrand-Geslin écrivait: «les ouvriers ont trouvé dans un lit de marne jaune pale [sic] 2 vertebres [sic] dont j'ai pu m'en procurer une $\mathrm{N}^{\circ} 13-3$ ». Cette vertèbre sera à rechercher dans les collections des sciences de la Terre du Muséum d'Histoire naturelle de Nantes, actuellement inaccessibles pour cause de déménagement (Denis Demarque, communication personnelle).

Dès la fin des années 1820, commença à être rapportée la présence de vertébrés fossiles dans les terrains jurassiques du Boulonnais (Rozet, 1827, p. 196, 200 ; Bertrand, 1829, p. 447 ; Brignon, 2015). Le 4 mars 1834, Eugène Robert (1806-1882) présenta, durant une séance de la Société géologique de France présidée par Constant Prévost, ses observations sur la géologie du Boulonnais et sur des ossements de «sauriens » qu'il avait découverts près de Boulogne-sur-Mer (Robert, 1834; Rozet, 1835, p. li ; Brignon, 2015, p. 58). Eugène Robert, de son nom complet Louis Eugène Robert, naquit et vécut longtemps à Meudon, dans l'actuel département des Hauts-de-Seine, commune dont il fut maire de 1870 à 1871. Géologue de formation, il effectua des voyages scientifiques, notamment dans le Nord de l'Europe. Il s'intéressa à la préhistoire, mais fut un fervent opposant à l'ancienneté de l'Homme et sa contemporanéité avec les espèces animales disparues de la grande faune froide (Hamy, 1882; Paillet, 2011). Il se retira vers la fin de sa vie à Sézanne dans la Marne où il entreprit des études sur la géologie et la paléontologie de cette région. Dans les éboulements de la falaise entre le port de Boulogne-sur-Mer et Le Portel, Eugène Robert avait récolté en automne 1833 de nombreux ossements fossiles dont une «dent, admirablement conservée, de Megalosaurus » qui devait avoir selon lui au moins $80 \mathrm{~mm}$ de hauteur. Il découvrit également plusieurs os de grandes dimensions et une dent dont la couronne usée lui rappelait celles que «Cuvier rapporte à des reptiles qui les usaient par la détrition comme les ruminants », faisant certainement allusion aux dents d'Iguanodon signalées dans le Sussex par Gideon Mantell (1790-1852) dans les années 1820 (Cuvier, 1824, p. 351 ; Taquet, 1984 ; Buffetaut, 1999). Il est ainsi possible qu'Eugène Robert ait eu entre les mains des dents d'un théropode et d'un dinosaure herbivore ainsi que des os de dinosaures du Jurassique supérieur du Boulonnais. Mais comme pour la vertèbre de Bertrand-Geslin, ces identifications restent hypothétiques, en l'absence d'illustrations et de renseignements plus précis.

Entre le 8 et le 13 septembre 1839, la Société géologique de France organisa une réunion extraordinaire à Boulogne-surMer. L'adjoint au maire de la ville, Dutertre-Delporte (17881862), avait personnellement œuvré pour mettre l'hôtel de ville à la disposition de cette illustre assemblée, qui réunissait Constant Prévost, président de la Société, et quelques sommités britanniques comme William Buckland, alors président de la Geological Society à Londres, Roderick Impey Murchison (1792-1871) et William Henry Fitton (1780-1861). Durant la séance du 9 septembre 1839, Prévost (1839; Sauvage, 1874, p. 18) présenta un fragment d'os de grande dimension trouvé par Dutertre-Delporte à La Poterie, commune de Wimille, dans le «calcaire portlandien» (Tithonien), que Buckland supposa être la partie supérieure d'un humérus ou d'un fémur. Quarante-et-un ans plus tard, alors que la Société géologique de France se réunissait à nouveau à Boulogne-sur-Mer, le paléontologue Harry Govier Seeley (1839-1909), spécialiste des reptiles fossiles, s'intéressa à son tour à ce fragment qui avait depuis été déposé au Musée de Boulogne-sur-Mer (Sauvage, 1874). Dans une courte note accompagnée d'une figure (Seeley, 1880), il le regarda comme l'extrémité distale d'un fémur de dinosaurien d'un nouveau genre. Ce spécimen correspond probablement à un fragment de radius de sauropode (Buffetaut et Martin, 1993; Buffetaut, 1995, p. 78). Notons qu'en 1857, Jacques Boucher de Pertes (1788-1868) dans ces Antiquités celtiques et antédiluviennes, faisait allusion à un autre os fossile que lui avait communiqué Dutertre-Delporte (Boucher de Pertes, 1857, p. 159). Ce spécimen, supposé être une portion d'omoplate « d'un individu monstrueux du genre saurien », fut trouvé « dans un banc de gryphea virgula (kimmeridge clay)»(Kimméridgien) entre le fort de la Crèche et l'ancien fort de Croï (orthographié parfois Croy, Croÿ ou Crouy) à Wimereux.

Dutertre-Delporte, de son nom de baptême Pierre Nicolas Maurice Dutertre, naquit à Boulogne-sur-mer, paroisse SaintJoseph, le 18 août 1788, fils de Marie Jeanne Emilie Testu, originaire de Chaltrait dans la Marne, et de Pierre Daniel Dutertre du Huitbois, avocat en Parlement et au Présidial de Boulogne-sur-Mer, adjoint au maire et notaire (ADPC, Boulogne-sur-Mer, Paroisse Saint-Joseph, baptêmes, mariages, sépultures, 1748-1790, 5 MIR 160/10/2). Le 17 juillet 1816, il épousa Julie Delporte, née le 4 floréal an II (23 avril 1794) (ADPC, Boulogne-sur-Mer, mariages, 1813-1827, 5 MIR 160/34). Après des études de droit, il devint commissaire-priseur à Boulogne. Il fut aussi adjoint au maire, capitaine de la Garde Nationale et fut nommé chevalier de l'Ordre Impérial la Légion d'Honneur. Il décéda le 20 décembre 1864 (ADPC, Boulogne-sur-Mer, décès, 1857-1865, 5 MIR 160/52). 
Dutertre-Delporte ainsi que Nicolas-Robert Bouchard (18021864), dit Bouchard-Chantereaux, un commerçant de Boulogne-sur-Mer, sont connus pour avoir formé jusque dans les années 1860 , les deux plus importantes collections de fossiles du Boulonnais (Sauvage, 1868; Brignon, 2015). Une grande partie de ces collections fut léguée au Musée d'Histoire naturelle de Boulogne-sur-Mer. Plusieurs restes de dinosaures de la collection Dutertre-Delporte y étaient répertoriés (Vadet et Rose, 1986) avant la dispersion des collections du musée en 2003 (Oudoire et al., 2014, p. 180). Le fragment d'os de sauropode de la collection Dutertre-Delporte signalé par Constant Prévost en 1839 était classiquement considéré comme le premier reste de dinosaure découvert dans le Jurassique supérieur du Boulonnais (Buffetaut et al., 1991; Buffetaut et Martin, 1993; Buffetaut, 1995).

L'étude de la correspondance du géologue Jules Desnoyers (1800-1887), conservée à la Bibliothèque nationale de France, a permis de découvrir fortuitement une lettre de Bruno Marmin (1782-1866) qui atteste qu'une dent de dinosaure avait été trouvée à Boulogne-sur-Mer dès 1835 (Annexe 1). Issu d'une ancienne famille du Nord de la France (Annexe 2), Marmin rentra, comme son père l'avait fait avant lui, dans l'administration des postes. Il débuta une partie de sa carrière aux PaysBas, qui avaient été annexés en 1810 à l'Empire français. En 1813, Bruno Marmin était « chef de correspondance des postes d'Amsterdam » (UA, BS Huwelijk, archive 481, 272-03, acte de mariage $\mathrm{n}^{\circ} 37,24$ mars 1813). Il fut ensuite nommé inspecteur des postes à Lille (Demeunynck et Devaux, 1832, p. 281). Passionné d'histoire naturelle, il constitua une collection de coquillages et de fossiles qui jouissait d'une bonne réputation et était souvent citée dans la littérature scientifique de l'époque (Desmarest, 1824; Blainville, 1825, p. 658 ; 1827, p. 81, 87, 100, 118-120, 122, 136; Bruguière et al., 1830, p. 400 ; Guérin, 1830 ; Des Moulins, 1837, p. 159, 163, 207). Amateur éclairé, il était en relation avec de nombreux géologues, conchyliologistes et malacologistes et n'hésitait pas à leur prêter ou à leur céder des spécimens pour qu'ils les étudiassent [Bruguière et al., 1830, p. 34, 91; Deshayes, 1831, p. 192 ; Bibliothèque municipale d'Avignon (Livrée Ceccano), Aut.6365-6366, lettres de Bruno Marmin à Marcel de Serres et Polydore Roux]. Plusieurs espèces de mollusques et d'échinodermes furent ainsi nommées en son honneur (Sherborn, 1928, p. 3893). Après la perte de son emploi, Marmin retourna s'installer en 1833 à Boulogne-sur-Mer, sa ville natale (Annexe 1). Il perdit à la même époque son épouse, Anne Joséphine (1788-1832), son frère unique, Alexandre (17801830 ), et deux amis proches. Accablé par ces malheurs, il tenta d'oublier sa douleur et de reprendre goût à la vie en se dévouant tout entier à ses collections de coquillages, de coquilles terrestres et fluviatiles, de fossiles et de monnaies anciennes. Il entreprit surtout de former une collection de «tous les débris fossiles » de l'arrondissement de Boulognesur-Mer et avait déjà réuni en l'espace de deux ans de nombreux brachiopodes et mollusques (bivalves, céphalopodes) des terrains mésozoïques de la région (Annexe 1). Marmin écrivait à Desnoyers pour lui demander de lui procurer des fossiles, des pièces de monnaies ou des brochures scientifiques. Jules Pierre François Stanislas Desnoyers, originaire de Nogent-le-Rotrou, était un membre actif et influent de la Société d'Histoire naturelle de Paris et de la Société géologique de France. Il était rentré en 1833 au Muséum
d'Histoire naturelle à Paris comme aide-naturaliste de géologie puis y avait été nommé bibliothécaire en chef. Marmin proposait en échange de mettre à sa disposition, ainsi qu'à celle d'Henri Marie Ducrotay de Blainville (1777-1850), sa collection de fossiles du Boulonnais et de leur offrir les doubles des fossiles qu'il possédait. Marmin avait apparemment des griefs contre plusieurs naturalistes parisiens comme Jean-Louis-Hardouin Michelin (1786-1867), Félix de Roissy (1771-1843), ou encore Alcide d'Orbigny (1802-1857), qui ne daignaient pas répondre à ses lettres, et qui, d'après lui, le regardaient « comme un homme mort ou inutile à leurs yeux » depuis qu'il avait quitté la capitale.

Dans sa lettre, Marmin signale également à Desnoyers qu'un de ces amis de Boulogne-sur-Mer, probablement Dutertre-Delporte, possédait «une collection considérable d'ossemens fossiles de grands sauriens ». Marmin s'était luimême procuré une grande dent de «Megalosaurus» dont il envoya un croquis à Desnoyers (Fig. 2). Un anglais, dont l'identité n'est pas dévoilée dans la lettre de Marmin, déclarait que cette dent était si rare qu'elle valait plus qu'une «dent d'or». La dent, dessinée grandeur nature par Marmin, a une hauteur de $110 \mathrm{~mm}$ et est brisée à deux endroits. Elle correspond à la dent figurée par Sauvage (1874, pl. 5, fig. 1) et Buffetaut et Martin (1993, pl. 1, Fig. A-B ; Buffetaut, 1995 , p. 81 , en haut à gauche). Sauvage $(1874$, p. 10$)$ indiqua également qu'elle avait été trouvée par Bruno Marmin et qu'elle provenait du « tuf du Fort de la Crèche vers la Tour de Croÿ (Portlandien à Perna Bouchardi) [sic] ». Cette formation correspond aux Assises de Croï du Tithonien (Geyssant et al., 1993). Sauvage assigna la dent de Marmin à Megalosaurus insignis Eudes-Deslongchamps et Lennier in Lennier, 1870. Cette espèce fut créée à partir d'une dent isolée du Kimméridgien inférieur du Cap de la Hève (Valenciennes, 1863 , p. 321 ; Lennier, 1870 , p. 35-36 ; 1887, pl. 1, fig. 1-3), dent qui était conservée au Musée d'histoire naturelle du Havre avant sa destruction sous les bombardements de 1944 (Cousin et Breton, 1994). Le peu de caractères différenciant les dents de théropodes, cette espèce est considérée comme un nomen dubium (Powell, 1987; Buffetaut et al., 1991; Buffetaut et Martin, 1993; Allain, 2002; Martill et al., 2006). La dent rapportée par Marmin (Theropoda indéterminé) n'en reste pas moins un spécimen remarquable par sa taille et son importance historique puisqu'elle représente de manière irréfutable le premier reste de dinosaure trouvé dans le Jurassique du Boulonnais. La lettre de Marmin à Desnoyers présentée ici prouve en effet que cette découverte est antérieure au 30 juillet 1835. Elle fut donnée par Marmin au Musée d'Histoire naturelle de Boulogne-sur-Mer où elle y était conservée avec le numéro d'inventaire MHNB 122 (ancien numéro 309) (Vadet et Rose, 1986, p. 86). Cette dent n'a malheureusement pas pu être retrouvée au Musée d'Histoire naturelle de Lille où une grande partie de la collection paléontologique de Boulogne-sur-Mer est mise en dépôt (Thierry Oudoire, communication personnelle).

\section{Les premiers dinosaures trouvés dans le crétacé}

En 1839, Henri Coquand (1813-1881) mentionna dans une note sur la géologie de la région d'Aix-en-Provence la découverte de deux fémurs de mastodonte dans la vallée du Val 


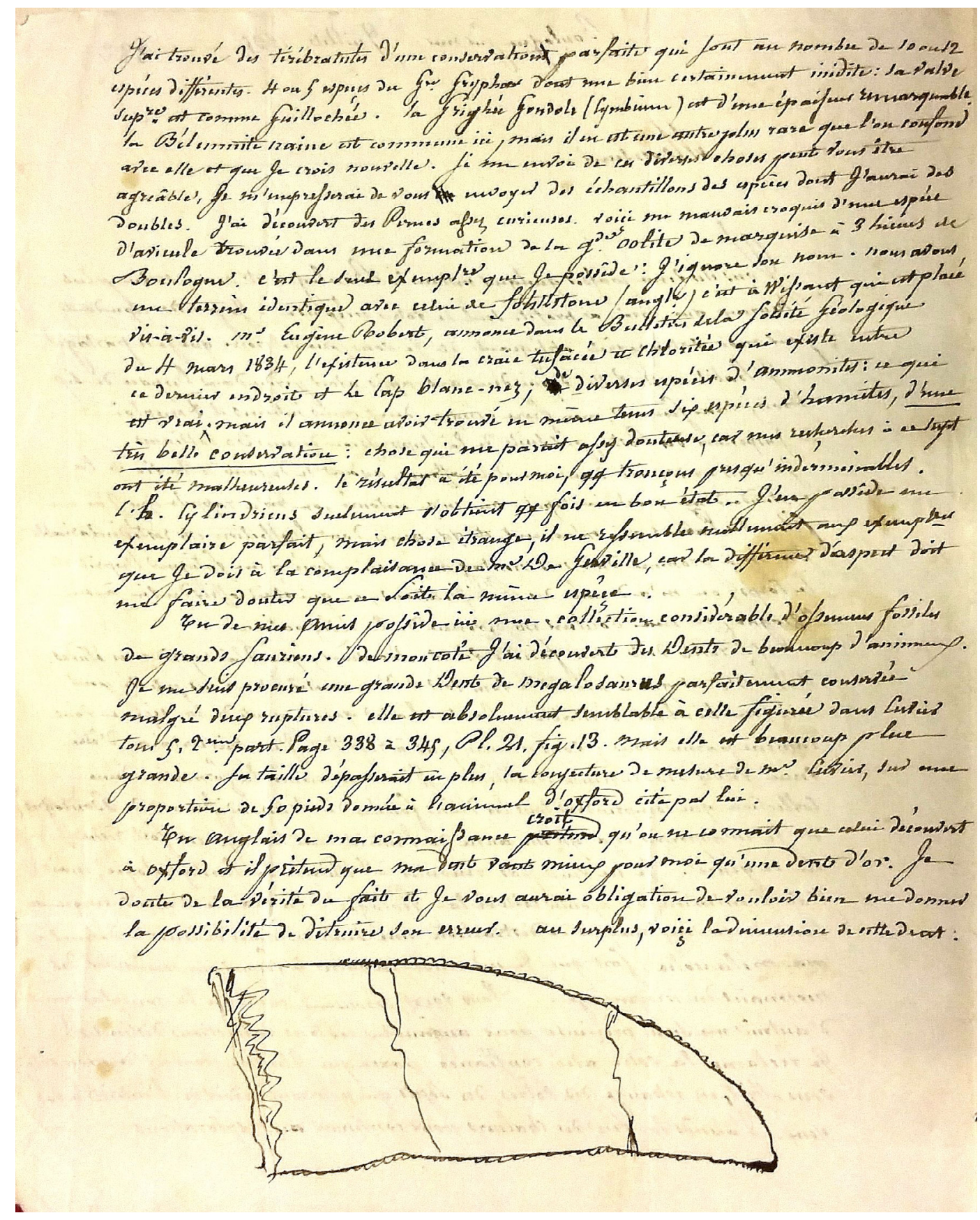

Fig. 2. Lettre inédite de Bruno Marmin à Jules Desnoyers (BNF NAF 24251/folio 282 verso) envoyée en juillet 1835 (cachet de la poste datée du 30 juillet 1835). Le croquis représente une dent de théropode de $110 \mathrm{~mm}$ de hauteur (grandeur naturelle dans le document original). Ce spécimen (MHNB 122) fut donné par Marmin au Musée d'Histoire naturelle de Boulogne-sur-Mer et fut identifié comme une dent de Megalosaurus insignis par Sauvage (1874). Document reproduit avec la permission de la Bibliothèque nationale de France.

Fig. 2. Unpublished letter by Bruno Marmin to Jules Desnoyers (BNF NAF 24251/folio 282 verso), postmark dated 30 July 1835. The sketch depicts a theropod tooth with a height of $110 \mathrm{~mm}$ (in natural size in the original document). This specimen (MHNB 122) was given by Marmin to the Musée d'Histoire naturelle de Boulogne-sur-Mer and was identified as a tooth of Megalosaurus insignis by Sauvage (1874). Reproduced by permission of the Bibliothèque nationale de France, Paris.

(Coquand, 1839, p. 78 ; Coquand, 1840, p. 210 ; VilleneuveFlayosc, 1856, p. 212). Ces os avaient une hauteur d'environ $70 \mathrm{~cm}$ et un diamètre allant jusqu'à $25 \mathrm{~cm}$. Coquand indiquait qu'ils avaient été trouvés près d'une propriété appartenant à $\mathrm{M}$. Desmichels. Les registres d'état civil des archives départe- mentales du Var montrent qu'une famille Desmichels était en effet implantée à cette époque au Val, un village situé dans la vallée de la Ribeirotte, au nord de Brignoles. On trouve notamment un certain Jean Chrisosthome Louis Étienne Desmichels, propriétaire et avocat, décédé le 14 août 1849 à 
l'âge de 72 ans. Un chirurgien de l'armée, Théophile-Marie Desmichels, avait également rédigé une thèse en 1828 intitulée «Esquisse de la topographie physique et médicale du Val (département du Var)» (Desmichels, 1828). Compte tenu de la présence de formations crétacées aux alentours du Val (Durand et al., 1979) et en particulier de formations fluviatiles gréseuses du Bégudien, il a été supposé que ces «fémurs» pussent être des os de dinosaures, et donc potentiellement les premiers dinosaures découverts dans le Crétacé supérieur européen (Buffetaut et al., 1993; Le Loeuff, 2001; Tortosa, 2014, p. 223). En l'absence d'autres renseignements, l'attribution de ces os à des dinosaures reste encore une fois hypothétique. Ayant déjà été mentionnées dans la littérature, nous ne détaillerons pas ici les découvertes d'ossements de grands sauriens attribuables à des dinosaures signalés en 1842 dans le Crétacé supérieur de Fox-Amphoux, par Ferdinand Panescorse (1808-1888) et Jean Dominique Doublier (1786-1874) (Doublier, 1842; Villeneuve-Flayosc, 1856, p. 227-228; Buffetaut et al., 1993; Le Loeuff, 2001, 2012; Lebrun et al., 2017), ni les découvertes de dinosaures faites par Philippe Matheron (1807-1899) à partir de 1846 dans le Crétacé provençal (Taquet, 2001).

Revenons, en revanche, sur une découverte bien connue qui s'avère être plus ancienne que ce que l'on croyait. Paul Gervais (1816-1879) décrivit et figura dans la première édition de sa Zoologie et Paléontologie française un humérus de sauropode trouvé dans le grès vert (Albien) près de Bédoin au pied du mont Ventoux (Vaucluse) (Gervais, 1848-1852, vol. 1, p. 263 ; vol. 3, pl. 63, fig. 3-4). Sur la base de ce spécimen, il créa un nouveau genre et une nouvelle espèce, Aepisaurus elephantinus. Dans la seconde édition de cet ouvrage, Gervais (1859, p. 464) lui-même changea le nom générique en «Aepysaurus», ce qui introduisit une confusion qui perdure encore aujourd'hui sur l'orthographe du nom. Selon l'article 33.3 du Code International de Nomenclature Zoologique (ICZN, 1999), Aepysaurus est une orthographe subséquente incorrecte (Steel, 1970). Mais revenons à l'histoire de cette découverte que Gervais attribuait à un certain Reynaux, d'Avignon. Son nom est en réalité Joseph-Prosper Renaux (1793-1852), connu sous le nom de Prosper Renaux (Fig. 3) (Crégut-Bonnoure, 2007). Le père de ce dernier, François Joseph Renaux (1752-1824), était originaire de Roubaix dans le département Nord et était venu s'installer à Alès en 1783 pour prendre la direction des houillères du secteur de Rochebelle dont Pierre François Pierre Tubeuf (1730-1795) avait la concession (Pérès, 1853). C'est à Alès que François Joseph Renaux fit connaissance d'Éléonore Pérès qu'il épousa en 1793. De cette union naquirent Prosper Renaux et un second fils, Jules Renaux. Prosper fit ses études à Nîmes et après le lycée, qu'il quitta en 1811, entra en qualité de conducteur dans les ponts et chaussées. Sous les ordres de différents ingénieurs, il fut chargé de la surveillance et du contrôle de plusieurs travaux, comme la rectification de la route d'Alès à La Voultesur-Rhône et la construction du pont de l'Hérault, entre Le Vigan et Valleraugue dans le Gard. À partir de 1818, il fut directement employé par la ville d'Alès pour diriger les travaux d'aménagements de la commune. Il postula ensuite pour la place d'architecte du département de Vaucluse, qui était vacante, et obtint sa nomination en avril 1825 . C'est ainsi qu'il alla s'établir à Avignon. Il profitait de toutes les occasions que lui offrait l'exercice de ses fonctions pour découvrir des

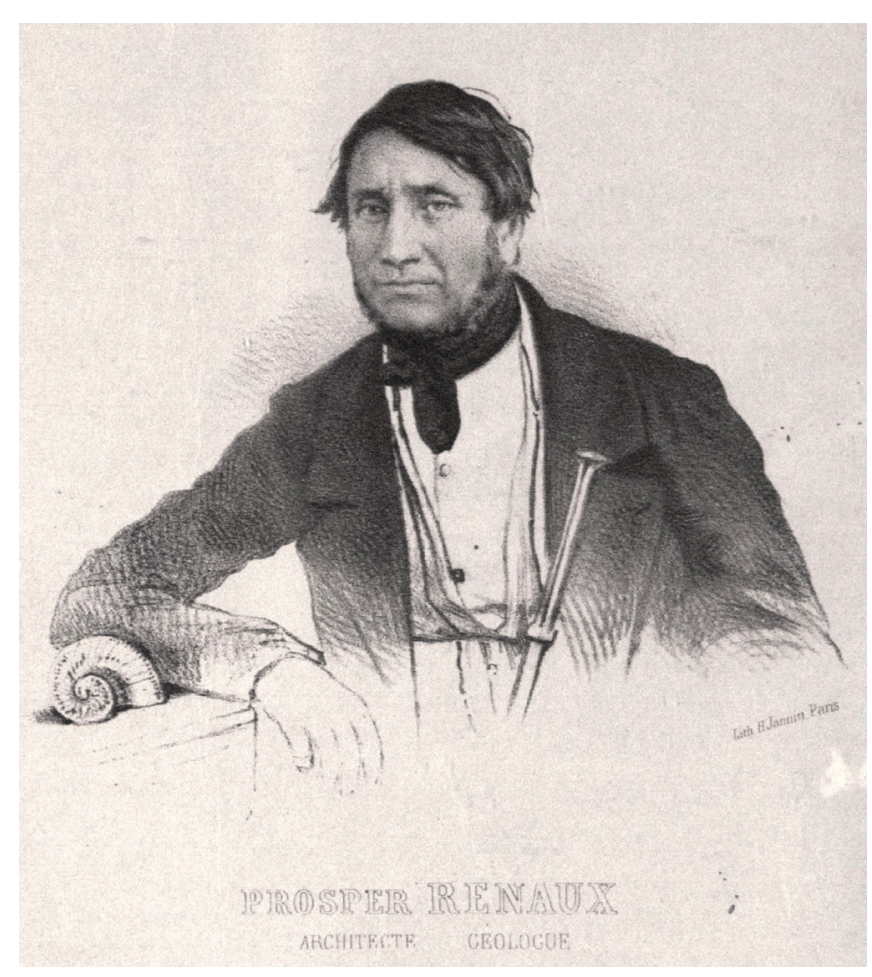

Fig. 3. Portrait de Prosper Renaux (1793-1852) publié par EmileJacques Pérès (1853). Dans l'état actuel des connaissances, Renaux est le premier à avoir découvert et étudié un dinosaure, authentifié de manière incontestable, dans le Crétacé provençal, et ce dès 1841. Photographie Bibliothèque municipale d'Avignon (Livrée Ceccano). Fig. 3. Portrait of Prosper Renaux (1793-1852) published by EmileJacques Pérès (1853). On the basis of current knowledge, Renaux was the first to discover and study an authenticated dinosaur remain from the Cretaceous of Provence, in 1841. Courtesy of the Bibliothèque municipale d'Avignon (Livrée Ceccano).

vestiges archéologiques. Il rédigea un catalogue, resté sous forme manuscrite, des monuments antiques du département de Vaucluse et contribua activement à la restauration de plusieurs d'entre eux. Ses travaux lui valurent d'être nommé membre de la Société française des monuments historiques en juin 1842 et membre correspondant du ministre de l'Intérieur pour la conservation des monuments historiques l'année suivante.

Le goût de son père pour la minéralogie influença certainement Prosper Renaux qui voua également une passion pour la géologie et les fossiles. Sa collection paléontologique était réputée (Raspail, 1842, p. 55-56) et est maintes fois citée par d'Orbigny dans sa Paléontologie française (voir par exemple Orbigny, 1840-1841, 1842-1843, 1842-1849, 18431847). Renaux déclara qu'il avait commencé à ramasser sérieusement les fossiles depuis 1824 (Annexe 3) mais, dès 1821, il avait déjà découvert au Mas d'Adger, entre Vézénobres et Saint-Hilaire-de-Brethmas (Gard), des vertèbres, vraisemblablement de reptiles, dans des «marnes schisteuses » gris bleuâtre datant probablement du Valanginien (Hombres-Firmas, 1838, p. 134). À partir de 1830, il entreprit l'étude approfondie de la géologie dont il se disait lui-même un « ardent amateur» (Annexe 3). Il avait ainsi parcouru à pied le département de Vaucluse et une bonne partie des départements voisins. Durant ses excursions, il avait recueilli un grand 
nombre de notes et de croquis sur la géologie de cette région. Il découvrit de nombreux gisements de fossiles dont la liste est donnée dans la biographie d'Emile-Jacques Pérès (1853). Renaux fut reçu membre de la Société géologique de France en septembre 1841. L'année suivante, lors de la réunion extraordinaire de la Société à Aix-en-Provence, il présenta ses observations sur la géologie des environs de Rustrel et de Gargas (Renaux, 1842).

Prosper Renaux envoya une lettre à Henri Marie Ducrotay de Blainville le 13 août 1842 pour l'informer qu'il avait découvert un os fossile à Bédoin, au pied du mont Ventoux (Annexe 3). Il joignait à son envoi une lithographie inédite qui a pu être retrouvée dans le fonds de Blainville conservé à la bibliothèque centrale du MNHN (Fig. 4). On y reconnaît l'humérus de sauropode qui servit plus tard d'holotype à
l'Aepisaurus elephantinus. Renaux indiquait également qu'il était sur le point d'envoyer au Muséum d'Histoire naturelle de Paris une collection de fossiles et de roches du Vaucluse. Une première caisse contenait des restes de mammifères des environs de Gargas (Eocène) et de Cucuron (Miocène) ainsi que des coquilles fossiles répartis en 235 lots destinés aux collections d'ostéologie et de conchyliologie du Muséum [BCM Ms BLA 12(1)]. Des échantillons de roches du Vaucluse ainsi que quelques fossiles «du grés vert, des marnes et des roches néocomiennes » et de «l'oxford-clay » étaient contenus dans deux autres caisses et étaient quant à eux destinés à la section de géologie du Muséum dirigée par Louis Cordier (1777-1861).

Renaux avait lui-même exécuté les dessins qui servirent à la réalisation de la lithographie représentant l'humérus de

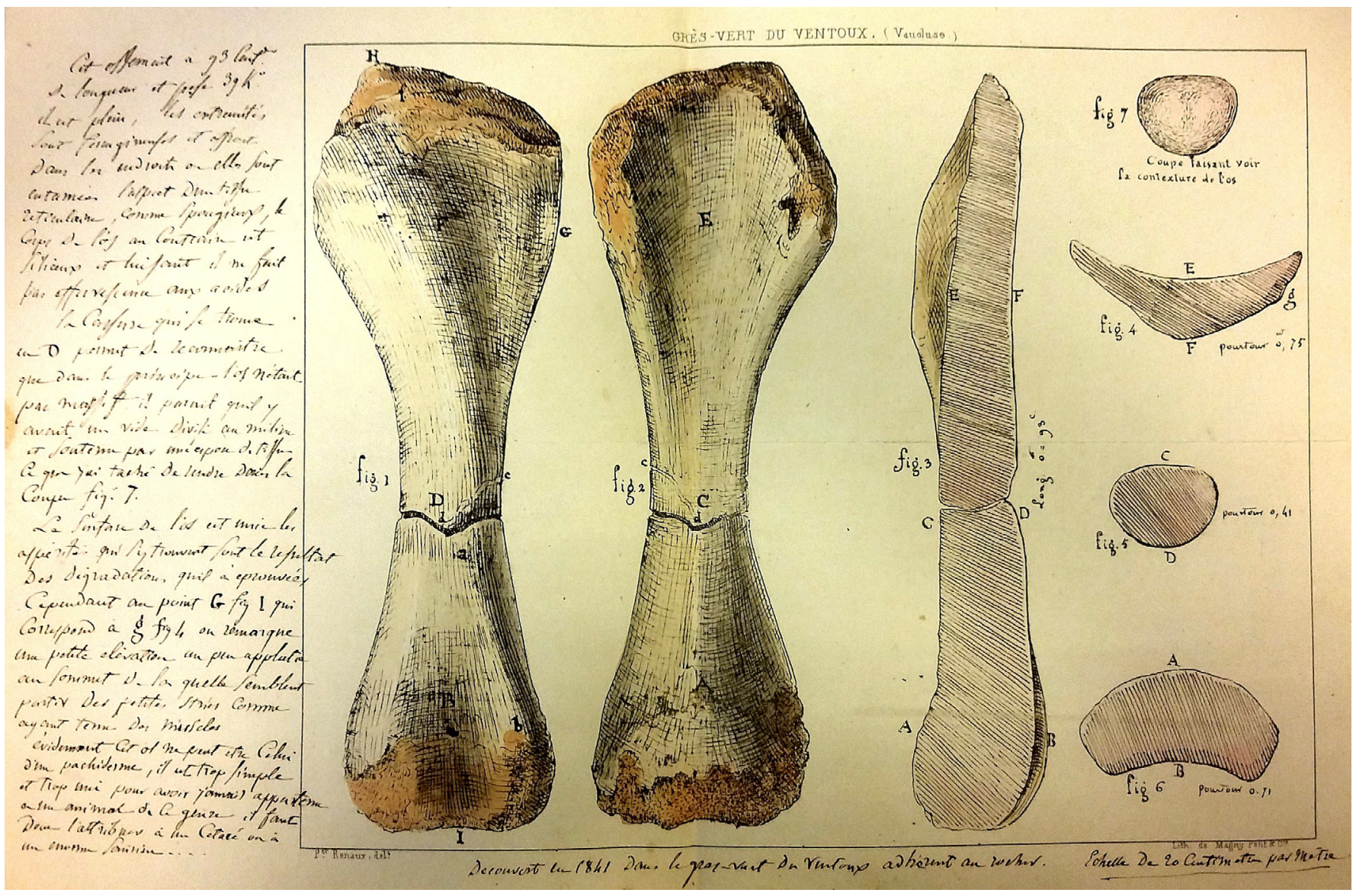

Fig. 4. Lithographie inédite, coloriée à la main, exécutée par l'imprimerie lithographique Magny, Petit et compagnie à Avignon d'après des dessins du géologue et architecte Prosper Renaux [BCM BLA 12(1)]. Cette planche représente un humérus de sauropode découvert par ce dernier dans le Grès Vert (Albien) du mont Ventoux (Vaucluse). Les notes dans les marges de la feuille, de la main de Renaux, décrivent le spécimen et indiquent qu'il fut trouvé en 1841. Renaux envoya ce document à Henri Marie Ducrotay de Blainville le 13 août 1842 . Le spécimen représenté servit d'holotype à l'Aepisaurus elephantinus décrit par Paul Gervais en 1852. (C) Muséum national d'Histoire naturelle (Paris) - Direction des bibliothèques et de la documentation.

Fig. 4. Unpublished hand-coloured lithographed plate from the typographic and lithographic firm Magny, Petit et compagnie, in Avignon. The plate was made from original drawings by the geologist and architect Prosper Renaux [BCM BLA 12(1)]. It depicts a sauropod humerus discovered by Renaux in the Grès Vert (Albian) of Mont Ventoux (Vaucluse). The handwritten notes by Renaux, in the margin of the plates, give a description of the specimen and indicate that it was found in 1841. Renaux sent this document to Henri Marie Ducrotay de Blainville on August 13, 1842. This humerus is the holotype of Aepisaurus elephantinus described by Paul Gervais in 1852. @) Muséum national d'Histoire naturelle (Paris)-Direction des bibliothèques et de la documentation. 
l'Aepisaurus elephantinus. Des notes de sa main dans les marges de la planche indiquent que l'os fut trouvé «en 1841 dans le grès vert du Ventoux adhérant au rocher ». Se référant aux figures de la planche, Renaux entreprend également la description du spécimen en ces termes: «Cet ossement a 93 cent. de longueur et pèse $39 \mathrm{k}^{0}[\mathrm{~kg}]$. Il est plein, les extrémités sont ferrugineuses et offrent dans les endroits où elles sont entamées l'aspect d'un tissu réticulaire, comme spongieux, le corps de l'os au contraire est siliceux et luisant. Il ne fait pas effervescence aux acides. La cassure qui se trouve en D permet de reconnaître que dans le principe, l'os n'était pas massif. Il paraît qu'il y avait un vide divisé au milieu et soutenu par une espèce de tissu, ce que j'ai taché de rendre dans la coupe fig. 7. La surface de l'os est unie. Les aspérités qui s'y trouvent sont le résultat des dégradations qu'il a éprouvées. Cependant au point $\mathrm{G}$ fig. 1 qui correspond à $\mathrm{g}$ fig. 4 , on remarque une petite élévation un peu aplatie au sommet de laquelle semblent partir des petites stries comme ayant tenu des muscles ». Enfin, Prosper Renaux tente d'identifier cet os et conclut: «évidemment cet os ne peut être celui d'un pachiderme [sic], il est trop simple et trop uni pour avoir jamais appartenu à un animal de ce genre. Il faut donc l'attribuer à un cétacé ou à un énorme saurien ». Dans sa lettre du 13 août 1842, Renaux demandait à de Blainville de l'aider à identifier ce spécimen (Annexe 3). Le brouillon de la réponse du savant parisien rédigé de sa main à la suite de la lettre de Renaux permet de connaître son avis: "Monsieur, ce fossile sur lequel vous voulez bien me consulter, me semble être une fémur ou un humérus omoplate d'un grand saurien très probablement du genre Megalosaurus ou Iguanodon des auteurs anglais». Les ratures montrent que de Blainville hésitait sur la position anatomique de cet os qu'il semble avoir finalement identifié comme une omoplate. Il est intéressant de noter qu'il rapprochait ce spécimen de deux genres que Richard Owen venait tout juste, en avril 1842, de ranger parmi les Dinosauria.

Renaux avait fait réaliser la planche présentée sur la Figure 4 car il projetait de publier un ouvrage sur la géologie du mont Ventoux et en particulier une note sur cet os comme le révèle Eugène Raspail (1812-1888), neveu du célèbre homme politique François Raspail (1794-1878) et inventeur du Neustosaurus gigondarum, crocodylomorphe du Valanginien de Gigondas (Raspail, 1842, p. 5): «M. Prosper Renaux a découvert tout récemment, dans les grès verts de Bedoin, un os qui appartient à un animal d'une taille gigantesque. Cet observateur éclairé se propose de mettre en ordre et de publier, à la suite de la description de ce précieux morceau d'histoire naturelle, les nombreux matériaux qu'il ne cessait de recueillir depuis plusieurs années ». Ce travail ne vit finalement jamais le jour et la lithographie de la Figure 4 en est aujourd'hui le seul témoignage connu. Le géologue du Gard, Emilien Dumas, témoigne également que Renaux avait rédigé des carnets de voyage remplis de dessins de fossiles et de coupes géologiques. Dumas concluait: «tous ces documents épars et les nombreuses collections qu'il a laissées font vivement regretter que M. Renaux n'ait pas eu le temps de publier le fruit de ses nombreuses et intéressantes recherches » (Pérès, 1853).

La note de Raspail (1842) est une des premières publications faisant référence à l'humérus de sauropode découvert par Renaux. Le naturaliste Jean Crespon (17971857), dans sa Faune méridionale, mentionna également ce spécimen en écrivant: «on a découvert au Mont-Ventoux (Vaucluse), un humérus d'une grande espèce de cétacé dont on ignore le nom » (Crespon, 1844, p. 112). Durant la séance du 8 septembre 1844 du Congrès scientifique de France qui se tint à Nîmes, Prosper Renaux présenta à l'assemblée le fameux os que le zoologiste Nicolas Joly (1812-1885) identifia comme un humérus de cétacé et Jules Teissier, médecin à Anduze, comme une «clavicule de saurien» (Renaux, 1845). La première allusion de Paul Gervais à cet humérus remonte à 1848 dans un article sur les reptiles inséré dans le Dictionnaire universel d'histoire naturelle (Gervais, 1848, p. 55). Prosper Renaux en avait donné un moulage en plâtre à Paul Gervais pour la Faculté des sciences de Montpellier. En 1848, ce dernier classait alors pertinemment ce spécimen parmi les dinosauriens. Il le rangea ensuite dans la famille des Mosasauridae (Gervais, 1848-1852) pour enfin avouer ne pas pouvoir lui assigner une place précise (Gervais, 1853). La première édition de sa Zoologie et Paléontologie française parut en 20 livraisons entre 1848 et 1852 . Il existe encore aujourd'hui une incertitude sur les dates exactes des actes nomenclaturaux introduits dans cet ouvrage. Un indice permet cependant de dater précisément la date de parution de la livraison qui contient la description de l'humérus d'Aepisaurus elephantinus. Gervais (1848-1852, vol. 1, p. 263) précise en effet que le spécimen faisait partie de la collection de «feu Reynaux [sic], d'Avignon ». Prosper Renaux étant décédé le 11 août 1852 (Pérès, 1853), l'espèce a donc bien été introduite en 1852. Notons que la partie proximale de l'humérus d'Aepisaurus elephantinus a été redécouverte en 2006 dans les collections de l'Université de Montpellier (BED01) (Tortosa et Buffetaut, 2007). On peut raisonnablement supposer que cette pièce fut donnée par Renaux ou ses héritiers à Paul Gervais qui était en poste à la Faculté des sciences de Montpellier entre 1845 et 1865 . Un moulage en plâtre de l'humérus complet est également conservé aujourd'hui dans la galerie de paléontologie du MNHN (numéro 1868-242). Il fut offert au Muséum par Renaux comme le révèle sa lettre à Blainville (Annexe 3 ). D'après Le Loeuff (1993), la proportion de l'humérus de l'Aepisaurus elephantinus suggère des affinités avec les Camarasauridae ou les Titanosauridae. Le peu de caractères diagnostiques de son holotype fait qu'Aepisaurus elephantinus est considéré comme un nomen dubium par plusieurs auteurs (Le Loeuff, 1993; Upchurch et al., 2004; Le Loeuff et al., 2013). Cette pièce n'en reste pas moins un des restes de sauropode les plus importants connus dans la partie moyenne du Crétacé européen.

Paul Gervais avait également brièvement décrit et figuré deux fragments d'os provenant de la «craie-tuffeau» de la plaine de «Lisle», aux environs de Périgueux, qui étaient conservés au Muséum d'Histoire naturelle à Paris (Gervais, 1848-1852, vol. 1, p. 264 ; vol. 3, pl. 63, fig. 1-2). Il attribuait également ces restes au genre Aepisaurus. On peut reconnaître sur la planche publiée par Gervais une partie distale d'humérus et un fragment d'os d'un membre de sauropode indéterminé (Buffetaut et al., 1991, p. 33). Le lieu exact de ces découvertes reste ambigu, pouvant s'agir de la commune de Lisle à une petite vingtaine de kilomètres au nord-ouest de Périgueux ou bien de la rivière l'Isle (Odin et al., 2001). En 1821, Alexandre Brongniart avait déjà observé la «craie tufau [sic]» sur les coteaux « qui bordent la rivière de Lille [sic], depuis Périgueux jusqu'au lieu dit la Massoulie» (Brongniart, 1821). Les 
formations crayeuses du Crétacé supérieur qui affleurent dans cette région s'étendent du Coniacien au Campanien. Les restes de dinosaures présentés par Gervais ont été supposés provenir des calcaires crayo-marneux du Campanien (Buffetaut et al., 1991 ; Buffetaut, 1995, p. 104) ou des calcaires gris crayeux et glauconieux du Santonien (Platel et al., 1989, p. 29). Jusqu'à aujourd'hui aucun autre renseignement n'était connu sur ces restes de dinosaure. En réponse à la lettre de Prosper Renaux datée du 13 août 1842, Henri Marie Ducrotay de Blainville déclarait au sujet de l'humérus de sauropode du mont Ventoux : «il m'intéresse d'autant plus que l'année dernière il nous en a été envoyé un que je crois tout semblable, des environs de Périgueux provenant des terrains de craie, comme le vôtre » (BCM Ms BLA 9, lettre de Renaux et réponse de Blainville). Cette allusion indiquerait donc qu'au moins un des fragments d'os de sauropode de Périgueux était rentré au Muséum d'Histoire naturelle en 1841. Par ailleurs, le catalogue manuscrit «des ossements fossiles de vertébrés placés dans les galeries de géologie et minéralogie » du Muséum, rédigé en 1861, nous apprend que ces spécimens, classés sous l'intitulé «reptiles fossiles de la plaine de Lisle, près de Périgueux (Dordogne) » avaient été donnés par un certain Simon Bornet. Ces restes y sont décrits comme un «tibia? se rapportant à une très grande espèce » (MNHN.F.AC9580) et «quatre portions d'os longs non déterminés» (MNHN.F.AC9581 à 9584).

\section{Conclusion}

Les scientifiques et amateurs français du XVIII et du début du $\mathrm{XIX}^{\mathrm{e}}$ siècle ont donc indéniablement joué un rôle important dans l'histoire des toutes premières découvertes de dinosaures. Des acteurs jusque-là oubliés ou mal connus ont été mis en lumière, comme (par ordre chronologique) Charles Bertrand-Geslin, Frédéric de La Fresnaye, Eugène Robert, Bruno Marmin, Alexandre Frédéric Bourrienne, Pierre Nicolas Maurice Dutertre-Delporte et Prosper Renaux. Les données inédites présentées dans cet article permettent de compléter la liste (Annexe 4) des tout premiers restes de dinosaures trouvés en France et de préciser les dates et les circonstances de leurs découvertes.

\section{Abréviations}

$\begin{array}{ll}\text { ADC } & \begin{array}{l}\text { Archives Départementales du Calvados, Caen } \\ \text { Archives Départementales du Pas-de-Calais, } \\ \text { ADPC }\end{array} \\ \text { Bainville } \\ \text { Bibliothèque centrale du Muséum national } \\ \text { d'Histoire naturelle, Paris } \\ \text { BNF } & \begin{array}{l}\text { Bibliothèque nationale de France, Paris } \\ \text { International Commission on Zoological }\end{array} \\ \text { ICZN } & \text { Nomenclature } \\ \text { MHNB } & \text { Musée d'Histoire naturelle de Boulogne-sur- } \\ \text { MNHN } & \text { Mer } \\ \text { UA } & \text { Muséum national d'Histoire naturelle, Paris } \\ \text { UM } & \text { Het Utrecht Archief, Utrecht, Pays-Bas } \\ & \text { Université de Montpellier }\end{array}$

Remerciements. Je remercie la Bibliothèque centrale du Muséum national d'Histoire naturelle, Paris et la Bibliothèque nationale de France pour m'avoir accordé la permission de reproduire les documents qui illustrent cet article; la Bibliothèque municipale d'Avignon (Livrée Ceccano) pour la communication du portrait de Prosper Renaux et des photographies de lettres manuscrites; Ronan Allain (MNHN, Paris), Denis Demarque (Muséum d'histoire Naturelle de Nantes), Suzanne Jiquel (Institut des Sciences de l'Évolution, Université Montpellier) et Thierry Oudoire (Musée d'Histoire Naturelle de Lille) pour les renseignements qu'ils m'ont communiqués sur les collections dont ils ont la charge. Cet article a bénéficié de la relecture critique de Ronan Allain. Je tiens à lui exprimer toute ma reconnaissance.

\section{Références}

Allain R. 2001. Redescription de Streptospondylus altdorfensis, le dinosaure théropode de Cuvier, du Jurassique de Normandie. Geodiversitas 23: 349-367.

Allain R. 2002. Les Megalosauridae (Dinosauria, Theropoda): nouvelle découverte et révision systématique: implications phylogénétiques et paléobiogéographiques. Thèse de doctorat, Muséum national d'Histoire naturelle. Paris: x, 329 p.

Allain R, Chure DJ. 2002. Poekilopleuron bucklandii, the theropod dinosaur from the Middle Jurassic (Bathonian) of Normandy. Palaeontology 45(6): 1107-1121.

Anonyme. 1833. Première section, Sciences naturelles. I. Minéralogie, géologie et palæonthologie. In: Congrès Scientifiques de France, première session, tenue à Caen en juillet 1833. Nicétas Périaux: Rouen, pp. 27-45 [ce rapport fut certainement rédigé par J.-A. Eudes-Deslongchamps qui était secrétaire de la section d'histoire naturelle].

Anonyme. 1837a. Bulletin bibliographique [de la séance de l'Académie du 31 juillet 1837]. Comptes rendus hebdomadaire des séances de l'Académie des sciences 5: 123-124.

Anonyme. 1837b. New fossil species. Magazine of Popular Science, and Journal of the useful Arts 4: 228.

Arnoult E. 1837. Bibliographie, ouvrages nouveaux. L'Institut, Journal général des Sociétés et travaux scientifiques de la France et de l'étranger, Section I (sciences mathématiques, physiques et naturelles) 5: 221, (novembre 1837): 381-383.

Bacheley C. 1778. Notice des pétrifications \& autres faits d'histoire naturelle qui se trouvent le long [des] côtes du Pays d'Auge. In: Lépecq de la Clôture L, ed., Collection d'observations sur les maladies et constitutions épidémiques. Rouen: Imprimerie privilégiée, 357-359.

Benson RBJ. 2010. A description of Megalosaurus bucklandii (Dinosauria: Theropoda) from the Bathonian of the UK and the relationships of Middle Jurassic theropods. Zoological Journal of the Linnean Society 158: 882-935.

Benson RBJ, Barrett PM, Powell HP, Norman DB. 2008. The taxonomic status of Megalosaurus bucklandii (Dinosauria, Theropoda) from the Middle Jurassic of Oxfordshire, UK. Palaeontology 51(2): 419-424.

Bertrand PJB. 1829. Précis de l'histoire physique, civile et politique, de la ville de Boulogne-sur-Mer et de ses environs, depuis les Morins jusqu'en 1814; suivi de la topographie médicale, de considérations de l'hygiène publique, d'une analyse de l'histoire naturelle du Boulonnais, d'un traité sur les bains de mer, et d'une biographie des hommes distingués nés dans ce pays, tome 2 . Boulogne: iv, $672 \mathrm{p}$.

Bertrand-Geslin C. 1833. Vertèbre de Megalosaurus dans l'oolithe moyenne du canal de Belle-Croix près de La Rochelle. Bulletin de la Société géologique de France 3: 281. 
Bigot A. 1945. La destruction des collections et des bibliothèques scientifiques de Caen. Bulletin de la Société Linnéenne de Normandie, série 9, volume supplémentaire: 1-75.

Blainville HM Ducrotay de. 1825. Manuel de malacologie et de conchyliologie. Paris \& Strasbourg: F. G. Levrault, viii, 664 p.

Blainville HM Ducrotay de. 1827. Mémoire sur les Bélemnites, considérées zoologiquement et géologiquement. Paris \& Strasbourg: F. G. Levrault, vii, 136 p., 5 pl.

Boneham BFW, Wyatt RJ. 1993. The stratigraphical position of the Middle Jurassic (Bathonian) Stonesfield Slate of Stonesfield, Oxfordshire, UK. Proceedings of the Geologists' Association 104 (2): 123-136.

Boucher de Perthes J. 1857. Antiquités celtiques et antédiluviennes, tome 2. Paris: Treuttel \& Wurtz, iv, xvi, 511 p.

Boué A. 1834. Résumé des progrès des sciences géologiques pendant l'année 1833, tome 5. Société Géologique de France: Paris : Société Géologique de France, iv, vii, 506, 1 p.

Brignon A. 2013. L'étude inachevée d'Étienne Geoffroy Saint-Hilaire sur les crocodiles fossiles (Thalattosuchia) de Normandie à la lumière de documents inédits. Annales de Paléontologie 99: 169205.

Brignon A. 2014. Les premières découvertes de crocodiliens fossiles dans la Pierre de Caen (Bathonien, Normandie) au travers des archives de Georges Cuvier. Revue de Paléobiologie 33(2): 379 418.

Brignon A. 2015. Les débuts de la paléoichthyologie en Normandie et dans le Boulonnais. Fossiles: Revue française de Paléontologie 21: 43-62.

Brignon A. 2016a. L'abbé Bacheley et la découverte des premiers dinosaures et crocodiliens marins dans le Jurassique des Vaches Noires (Callovien/Oxfordien, Normandie). Comptes Rendus Palevol 15(5): 595-605.

Brignon A. 2016b. Le premier «chasseur de dinosaures » en France : l'abbé Charles Bacheley (1716-1795). Fossiles: Revue française de Paléontologie 27: 36-42.

Brongniart A. 1821. Sur les caractères zoologiques des formations, avec l'application de ces caractères à la détermination de quelques terrains de craie. Annales des Mines 6: 537-572, pl. 7-8.

Bruguière JG, Lamarck JB, Deshayes PG. 1830. Histoire naturelle des vers, tome 2. Paris: Veuve Agasse, vii, 256, 594 p.

Brockhaus FA. 1837. Französisches Literatur. Allgemeine Bibliographie für Deutschland 2 (37, 15 septembre 1837): 570-572.

Bronn HG. 1837. Lethae Geognostica, oder Abbildungen und Beschreibungen der für die Gebirgs-Formationen bezeichnendsten Versteinerungen, Erster Band. Stuttgart: E. Schweizerbart's Verlagshandlung, $544 \mathrm{p}$.

Buckland W. 1824. Notice on the Megalosaurus or great fossil lizard of Stonesfield. Transactions of the Geological Society, London, Series 2, 1(2): 390-396, pl. 40-44.

Buckland W. 1836. Geology and mineralogy considered with reference to natural theology. London: William Pickering, vol. 1: xvi, 599, (1) p.; vol. 2: vii, 128 p., 87 pl.

Buffetaut E. 1983. La paléontologie des vertébrés mésozoïques en Normandie du $18^{\mathrm{e}}$ siècle à nos jours : un essai historique. Actes $d u$ Muséum de Rouen 2: 39-59.

Buffetaut E. 1995. Dinosaures de France. Éditions BRGM, 144 p.

Buffetaut E. 1999. Mantell, Cuvier, Buckland and the identification of Iguanodon: a contribution based on unpublished annotations by Mantell. Oryctos 2: 101-109.

Buffetaut E. 2011. Chercheurs de dinosaures en Normandie. Louviers: Ysec, $160 \mathrm{p}$.

Buffetaut E, Cuny G, Le Loeuff J. 1991. French dinosaurs: the best record in Europe? Modern Geology 16: 17-42.
Buffetaut E, Cuny G, Le Loeuff J. 1993. The discovery of French dinosaurs. Modern Geology 18: 161-182.

Buffetaut E, Cuny G, Pharisat A. 1994. William Buckland's French Megalosaurus. Terra Nova 6(3): 323-325.

Buffetaut E, Martin M. 1993. Late Jurassic dinosaurs from the Boulonnais (Northern France): a review. Revue de Paléobiologie, volume special 7: 17-28.

Carrano MT, Benson RBJ, Sampson SD. 2012. The phylogeny of Tetanurae (Dinosauria: Theropoda). Journal of Systematic Palaeontology 10(2): 211-300.

Caumont A de. 1827. Extrait du rapport lu dans la séance publique du 24 mai 1826 sur les travaux de la Société Linnéenne de Normandie depuis le 24 mai 1825 jusqu'au 24 mai 1826, Société Linnéenne de Normandie, résumé des travaux pendant l'année 1835-1836. Mémoires de la Société Linnéenne de Normandie, années 1826 et 1827: xiv-xxxvii.

Caumont A de. 1828. Essai sur la topographie géognostique du département du Calvados. Mémoires de la Société Linnéenne de Normandie 1828: 59-366, pl. 1-7, 8 (partim).

Caumont A de. 1838. Extraits des rapports sur les travaux annuels de la Société, année 1833-1834; rapport lu à la séance publique tenue à Falaise, le 5 juin 1834. Mémoires de la Société Linnéenne de Normandie 6: (1834-1838), 1-8.

Coquand H. 1839. Lettre dans laquelle il cherche à prouver, contre l'opinion de M. Dufrénoy, que les gypses d'Aix qui constituent un étage supérieur à la molasse, sont contemporains de ceux de Montmartre. Bulletin de la Société géologique de France 10: 7782, pl. 2.

Coquand H. 1840. Cours de géologie, professé au Musée d'Histoire naturelle d'Aix. Aix: Imprimerie de Martin, 224 p. (La page de titre est datée 1839 alors que la couverture imprimée porte la date de 1840).

Cousin R, Breton G. 1994. Inventaire des spécimens de vertébrés fossiles conservés au Muséum d'Histoire naturelle du Havre et détruits lors des bombardements du 5 septembre 1944. Annales $d u$ Muséum du Havre 48: 1-10.

Crégut-Bonnoure É. 2007. Les pionniers de la géologie du Ventoux. In: Barruol G, Dautier N, Mondon B, eds. Le mont Ventoux, encyclopédie d'une montagne provençale. Forcalquier: Les Alpes de lumière, pp. 29-30.

Crespon J. 1844. Faune méridionale, ou description de tous les animaux vertébrés vivans et fossiles, sauvages ou domestiques qui se rencontrent toute l'année ou qui ne sont que de passage dans la plus grande partie du Midi de la France, suivie d'une méthode de taxidermie ou l'art d'empailler les oiseaux, tome 1. Montpellier : Chez l'auteur, Nîmes et chez M. Lebrun, xxviii, 320, (2) p.

Cressac E-B de, Manès G. 1830. Notice géognostique sur le bassin secondaire compris entre les terrains primitifs du Limousin et ceux intermédiaires de la Véndée. Annales des Mines 7(Série 2): 169-278.

Cuvier G. 1808. Sur les ossemens fossiles de crocodiles, et particulièrement sur ceux des environs du Havre et de Honfleur, avec des remarques sur les squelettes des sauriens de la Thuringe. Annales du Muséum d'Histoire Naturelle 12: 73-110.

Cuvier G. 1824. Recherches sur les ossemens fossiles, où l'on rétablit les caractères de plusieurs animaux dont les révolutions du globe ont détruit les espèces, $2^{\mathrm{e}}$ édition, tome $5,2^{\mathrm{e}}$ partie. Paris: G. Dufour et E. d'Ocagne, 547 p., 33 pl.

De la Beche HT. 1830a. Notes on the geographical distribution of organic remains contained in the oolitic series of the Great London and Paris Basin, and in the same series of the South of France. Philosophical Magazine 8(45, september 1830): 208-213.

De la Beche HT. 1830b. Geological notes. London: Treuttel and Würtz, Treuttel junior, and Richter, ii, 69, xlii p. 
De la Beche HT. 1831. A geological manual. London: Treuttel and Würtz, Treuttel junior, and Richter, xii, $535 \mathrm{p}$.

Dean DR. 1999. Gideon Mantell and the discovery of dinosaurs. Cambridge University Press: xix, 290 p.

Delair JB, Sarjeant WAS. 1975. The earliest discoveries of dinosaurs. Isis 66(1): 4-25.

Delair JB, Sarjeant WAS. 2002. The earliest discoveries of dinosaur bones: the records re-examined. Proceedings of the Geologists' Association, London 113(3): 185-197.

Demeunynck J, Devaux D. 1832. Annuaire statistique du département du Nord, 4 ${ }^{\mathrm{e}}$ année. Lille: L. Danel, 396 p.

Des Moulins C. 1837. Troisième mémoire sur les échinides, synonymie générale. Actes de la Société Linnéenne de Bordeaux 9(49): 45-364.

Deshayes GP. 1831. Description de coquilles caractéristiques des terrains. Paris \& Strasbourg: F. G. Levrault, vii, 264 p., 14 pl.

Desmarest AG. 1824. Note sur la nécessité de retirer le corps organisé nommé Amphitoïte, de la série des fossiles animaux. Annales des Sciences Naturelles 1: 331-334.

Desmichels T-M. 1828. Esquisse de la topographie physique et médicale du Val (département du Var). Paris : Didot le jeune, $33 \mathrm{p}$.

Dicquemare J.-F. 1776. Ostéolithes. Observations sur la Physique, sur l'Histoire Naturelle et sur les Arts 7: 406-414.

Doublier JD. 1842. Ossements de saurien trouvés dans le Var dans le terrain à lignites. Bulletin de la Société géologique de France 13: 449.

Dugué O, Dujardin L, Leroux P, Savary X. 2010. La Pierre de Caen, des dinosaures aux cathédrales. Caen : Musée de Normandie, $116 \mathrm{p}$.

Durand J-P, Guieu G, Mennessier G, Rouire J, avec la collaboration de Damiani L, Féraud J, Durozoy G. 1979. Notice explicative, Carte géologique de France (1/50000), feuille Brignoles (1022). Orléans: BRGM, 30 p.

Eudes-Deslongchamps J-A. 1836a. Plusieurs parties d'un mémoire sur un très-grand animal fossile découvert, l'an dernier, dans les carrières de la Maladrerie à un quart de lieu de Caen. In : Analyse des travaux de la Société pendant l'année académique 1835-1836, Séance Publique de la Société Linnéenne de Normandie tenue à Vire le 24 mai 1836. Caen: A. Hardel \& Paris : Dérache, pp. 14-25.

Eudes-Deslongchamps J-A. 1836b. Plusieurs parties d'un mémoire sur un très-grand animal fossile découvert en 1835 , dans les carrières de la Maladrerie, à un quart de lieue de Caen. In : Société Linnéenne de Normandie, résumé des travaux pendant l'année 1835-1836. L'Institut, Journal général des Sociétés et travaux scientifiques de la France et de l'étranger, Section I (sciences mathématiques, physiques et naturelles) 4 (176, 21 septembre 1836): 311-312.

Eudes-Deslongchamps J-A. 1837a. Mémoire sur le Poekilopleuron bucklandii, grand saurien fossile, intermédiaire entre les crocodiles et les lézards. Caen: A. Hardel, 114 p., 8 pl.

Eudes-Deslongchamps J-A. 1837b. Mémoire sur le Poekilopleuron bucklandii grand saurien fossile, intermédiaire entre les crocodiles et les lézards. Annales des Sciences Naturelles 7(série 2): 255-256.

Eudes-Deslongchamps J-A. 1838. Mémoire sur le Poekilopleuron bucklandii, grand saurien fossile, intermédiaire entre les crocodiles et les lézards. Mémoires de la Société Linnéenne de Normandie 6 (1834-1838): 37-146, 8 pl.

Farber PL. 1982. The emergence of ornithology as a scientific discipline: 1760-1850. Dordrecht: D. Reidel, xxi, 191 p.

Geoffroy Saint-Hilaire E. 1837. Des changements à la surface de la terre, qui paraissent dépendre originairement et nécessairement de la variation préexistante, incessante, lente et successive, des milieux ambiants divers et consécutifs, du Globe terrestre. Comptes rendus hebdomadaire des séances de l'Académie des sciences 5: 183-194.
Gervais P. 1848. Reptiles. In : d'Orbigny C, ed. Dictionnaire universel d'histoire naturelle, tome 11. Paris: Renard, Martinet et $\mathrm{C}^{\mathrm{ie}}$, pp. 1-65.

Gervais P. 1848-1852. Zoologie et paléontologie françaises (animaux vertébrés) ou nouvelles recherches sur les animaux vivants et fossiles de la France, tome 1. Paris : Arthus Bertrand, vol. 1 : iv, viii, 271 p.; vol. 2 : iv, $42,16,2,8,8,8,12,10,8,2,14,16$ p.; vol. 3 : iv p., 80 pl. Gervais P. 1853. Observations relatives aux reptiles fossiles de France (deuxième partie). Comptes rendus hebdomadaires des séances de l'Académie des sciences 36: 470-474.

Gervais P. 1859. Zoologie et paléontologie françaises, 2e édition. Paris : Arthus Bertrand, vol. 1 : viii, 544 p.; vol. 2 : xii p., 84 pl.

Gevril F. 1828. Cabinet d'histoire naturelle de Besançon. Annuaire Statistique et Historique du Département du Doubs 16: 461-463.

Geyssant JR, Vidier J-P, Herbin J-P, Proust J-N, Deconinck J-F. 1993. Biostratigraphie et paléoenvironnement des couches de passage Kimméridgien/Tithonien du Boulonnais (Pas-de-Calais) : nouvelles données paléontologiques (ammonites), organisation séquentielle et contenu en matière organique. Géologie de la France 4: $11-24$.

Gigot P, Dupret L, Le Gall J, avec la collaboration de Coutard J-P, Ozouf J-C, Pay T, Lecointe A, Desloges J, Deroin J-P. 1999. Notice explicative, Carte géologique de France (1/50 000), feuille Falaise (176). Orléans : BRGM, 154 p.

Guenard A. 1844. Besançon, description historique des monuments et établissements publics de cette ville. Besançon: Bintot, vi, 292 p., 5 pl.

Guérin FE. 1830. Magasin de conchyliologie, ou descriptions et figures de mollusques vivants et fossiles inédits ou non encore figurés, $3^{\mathrm{e}}$ livraison. Paris : Chez Lequien fils, 16 p., pl. 17-24.

Haigneré D. 1873. M. Charles Marmin. Bulletin de la Société Académique de l'Arrondissement de Boulogne-sur-Mer 2: 74-88.

Hamy E-T. 1882. Nécrologie - Eug. Robert. Revue d'Ethnographie 1: 461.

Hantzpergue P, Mazeau M, Dupuis J, Cariou E, Ducloux J. 1988. Notice explicative, Carte géologique de France (1/50 000), feuille Surgères (634). Orléans: BRGM, $19 \mathrm{p}$.

Hombres-Firmas LA. 1838. Recueil de mémoires et d'observations de physique, de météorologie, d'agriculture et d'histoire naturelle, partie 4, mémoires et observations d'histoire naturelle. Nîmes: Imprimerie Ballivet et Fabre, iv, 212, 4 p., 5 pl.

International Commission on Zoological Nomenclature (ICZN). 1999. International Code of Zoological Nomenclature, $4^{\mathrm{e}}$ édition. London: International Trust for Zoological Nomenclature, $306 \mathrm{p}$.

Lebrun P, Valentin X, Garcia G. 2017. Les pionniers en paléontologie: un aperçu des découvreurs et des découvertes en Provence. Fossiles : Revue française de Paléontologie 29: 5-12.

Lefèbvre A. 1908. La célèbre inconnue de Prosper Mérimée, sa vie et ses œuvres authentiques, avec documents, portraits et dessins inédits. Paris : E. Sansot, vi, 396, 1 p.

Le Loeuff J. 1993. European titanosaurids. Revue de Paléobiologie 7 (volume spécial): 105-117.

Le Loeuff J. 2001. Les dinosaures du Crétacé supérieur (CampanienMaastrichtien) d'Europe : une histoire des découvertes. In : Actas de las II Jornadas Internacionales sobre paleontología de Dinosaurios y su Entorno, Septembre 2001. Burgos: Salas de los Infantes, pp. 47-70.

Le Loeuff J. 2012. Ferdinand Panescorse and his classification of coprolites. In : Hunt AP, Milàn J, Lucas SG, Splielmann JA, eds. Vertebrate Coprolites. New Mexico Museum of Natural History and Science, Bulletin 57: 147-151.

Le Loeuff J, Suteethorn S, Buffetaut E. 2013. A new sauropod dinosaur from the Albian of Le Havre (Normandy, France). Oryctos 10: $23-30$. 
Lennier G. 1870. Études géologiques et paléontologiques sur l'embouchure de la Seine et les falaises de Haute Normandie. Le Havre: Imprimerie Eugène Costey, xvi, 245 p., 14 pl.

Lennier G. 1887. Études paléontologiques. Description des fossiles du Cap de la Hève. Bulletin de la Société géologique de Normandie 12(1886): 17-98, 22 pl.

Leonhard KC von, Bronn HG. 1837a. Eudes Deslongchamps hat bei der Line'schen Sozietät der Normandie im Jahre 1835-36 einen Vortrag gehalten über ein riesenmäsiges Reptil, Poecilopleuron, aus dem Kalke von Caen. Neues Jahrbuch für Mineralogie, Geognosie, Geologie und Petrefaktenkunde 1837: 99-101.

Leonhard KC von, Bronn HG. 1837b. Neueste Literatur. Neues Jahrbuch für Mineralogie, Geognosie, Geologie und Petrefaktenkunde 1837: 564-568.

Magnin A. 1910. Charles Nodier naturaliste, ses œuvres scientifiques publiées et inédites. Mémoires de la Société d'Émulation du Doubs 4 (Série 8, année 1909): 411-506.

Martill DM, Earland S, Naish D. 2006. Dinosaurs in marine strata: evidence from the British Jurassic, including a review of the allochthonous vertebrate assemblage from the marine Kimmeridge Clay Formation (Upper Jurassic) of Great Britain. In : Colectivo Arqueológico-Paleontológico Salense, ed. Actas de las III Jornadas sobre Dinosaurios y su Entorno 16-17 Sep. 2004. Burgos: Salas de los Infantes, pp. 35-72.

Meyer H von 1832. Palaeologica zur Geschichte der Erde und ihrer Geschöpfe. Frankfurt am Main: Siegmund Schmerber, xii, 560 p.

Moody RTJ, Buffetaut E, Naish D, Martill DM, eds. 2010. Dinosaurs and other extinct saurians: a historical perspective. Geological Society Special Publications 343. London: The Geological Society, vi, $394 \mathrm{p}$.

Odin GS, Buffetaut E, Le Loeuff J, Normand C, Platel J-P. 2001. Reptilian remains from the late Campanian in Tercis les Bains (Landes) and Vanxains (Dordogne). In: Odin GS, ed. The Campanian-Maastrichtian Stage Boundary, Developments in Palaeontology and Stratigraphy, vol. 19. Elsevier Science B.V.: pp. 665-74.

Orbigny A d'. 1840-1841. Paléontologie française. Description des mollusques et rayonnés fossiles. Terrains crétacés, tome 1, céphalopodes. Paris : Victor Masson, 662 p.

Orbigny A d'. 1842-1843. Paléontologie française. Description des mollusques et rayonnés fossiles. Terrains crétacés, tome 2, gastéropodes. Paris : Victor Masson, iv, 456 p.

Orbigny A d'. 1842-1849. Paléontologie française. Description des mollusques et rayonnés fossiles. Terrains jurassiques, tome 1, céphalopodes. Paris : Victor Masson, 642 p.

Orbigny A d'. 1843-1847. Paléontologie française. Description des mollusques et rayonnés fossiles. Terrains crétacés, tome 3, lamellibranches. Paris : Victor Masson, iv, $807 \mathrm{p}$.

Oudoire T, Cuvelier J, D'Andrea J, Koch C, Dhainaut A. 2014. Le département de géologie du Musée d'Histoire naturelle de Lille, des collections à l'image de l'histoire géologique régionale. Mémoires de la Société géologique du Nord 17: 175-183.

Owen R. 1842. Report on the British Reptiles, part 2. Report of the British Association for the Advancement of Science. 11th meeting, Plymouth, 1841: pp. 60-204.

Paillet P. 2011. Le mammouth de la Madeleine (Tursac, Dordogne) dans son siècle et aujourd'hui. Paleo 22: 223-270.

Pérès E-J. 1853. Notice biographique sur Joseph-Prosper Renaux, d'Alais [sic]. Alès: Imprimerie de veuve Veirun, 50 p., 1 portrait.

Pillet P. 1837. Livres français. Bibliographie de la France 26 (35, septembre 1837): 425-433.

Platel J-P, Célérier G, Duchadeau-Kervazo C, Charnet F, Chigot D. 1989. Notice explicative, Carte géologique de France (1/50 000), feuille Périgueux-Ouest (758). Orléans : BRGM, 82 p.
Powell HP. 1987. Megalosaurid dinosaur jawbone from the Kimmeridge Clay of the seabed of West Bay, Dorset. Proceedings of the Dorset Natural History and Archaeological Society 109: 105-108.

Prévost C. 1839. Fragment d'un os d'une grande dimension, dans le calcaire portlandien, près de Wimille. Bulletin de la Société géologique de France 10: 396.

Raspail E. 1842. Observations sur un nouveau genre de saurian fossile, le Neustosaurus gigondarum, n., avec quelques notes géologiques sur la commune de Gigondas. Paris: Meilhac \& Avignon: Clément St-Just, iv, 56 p., 1 pl.

Renaux P. 1842. Compte rendu de la course faite par la réunion géologique à Rustrel et à Gargas. Bulletin de la Société géologique de France 13: 497-506.

Renaux P. 1845. Sur un os énorme trouvé dans le grès vert près du Mont-Ventoux. Congrès scientifique de France 12: 119.

Robert E. 1834. Notes sur le Boulonnais, notamment sur ses ossemens fossiles, ses marbres et ceux de l'Irlande. Bulletin de la Société géologique de France 5: 310-317.

Rose J-M, Vadet A. 1986. Catalogue commenté des types de crocodiliens fossiles du Musée d'Histoire naturelle de Boulognesur-Mer. Mémoires de la Société Académique du Boulonnais 1 (2): 72-85, pl. 13-17.

Rothschild B, Tanke DH. 2005. Theropod paleopathology. In: Carpenter K, ed. The carnivorous dinosaurs. Bloomington \& Indianapolis: Indiana University Press, pp. 351-365.

Rozet E. 1827. Essai sur la constitution géognostique des environs de Boulogne-sur-Mer. Mémoires de la Société d'Histoire Naturelle de Paris 3: 182-208.

Rozet E. 1835. Résumé des travaux de la Société géologique de France, et des progrès de la géologie en France, depuis le $1^{\text {er }}$ novembre 1833, jusqu'au $1^{\mathrm{er}}$ novembre 1834. Bulletin de la Société géologique de France 6: i-xci.

Sauvage H-E. 1868. Catalogue des poissons des formations secondaires du Boulonnais. Mémoires de la Société Académique de l'Arrondissement de Boulogne-sur-Mer 2 (1), (1866-1867): 53-152, 4 pl.

Sauvage H-E. 1874. Mémoire sur les dinosauriens et les crocodiliens des terrains jurassiques de Boulogne-sur-Mer. Mémoires de la Société géologique de France 10(Série 2): 1-58, pl. 5-10.

Seeley H-G. 1880. Note sur l'extrémité distale d'un femur de dinosaurian, provenant du Portlandien supérieur de la Poterie, près de Boulogne, mentionné par M. C. Prévost à la réunion de la Société Géologique de France en 1839. Bulletin de la Société géologique de France 8(Série 3): 520-522.

Sharpe T, McCartney PJ. 1998. The papers of H.T. De la Beche (1796-1855) in the National Museum of Wales. National Museums of Wales, Geological Series 17: 1-257.

Sherborn CD. 1928. Index animalium, part 15. London: British Museum (Natural History), pp. 3747-3970.

Spalding DAE, Sarjeant WAS. 2012. Dinosaurs: the earliest discoveries. In: Brett-Surman MK, Holtz Jr. TR, Farlow JA, eds. The complete dinosaur. Bloomington \& Indianapolis: Indiana University Press, 3-23.

Steel R. 1970. Saurischia. Handbüch Handbuch der Paläoherpetologie, vol. 14. Stuttgart: Gustav Fischer, v, 87 p.

Sues H-D. 2012. European dinosaur hunters of the nineteenth and twentieth centuries. In : Brett-Surman MK, Holtz Jr. TR, Farlow JA, eds. The complete dinosaur. Bloomington \& Indianapolis: Indiana University Press, pp. 45-59.

Taquet P. 1984. Cuvier, Buckland, Mantell et les dinosaures. In : Buffetaut E, Mazin J-M, Salmon E, eds. Actes du symposium paléontologique Georges Cuvier. 25-28 octobre 1982. Montbéliard, pp. 475-494.

Taquet P. 1994. L'empreinte des dinosaures. Paris : Odile Jacob, 363, $1 \mathrm{p}$. 
Taquet P. 2001. Philippe Matheron et Paul Gervais : deux pionniers de la découverte et de l'étude des os et des œufs de dinosaures de Provence (France). Geodiversitas 23(4): 611-623.

Taquet P, Contini D. 1997. William Buckland et le «mégalosaure » de Franche-Comté. Nouvelles données historiques, stratigraphiques et paléogéographiques. Annales de Paléontologie (Vert. -Invert.) 83(1): 93-110.

Tortosa T. 2014. Vertébrés continentaux du Crétacé supérieur de Provence (Sud-Est de la France). Thèse de doctorat, Université Pierre et Marie Curie. Paris: 6, 461 p.

Tortosa T, Buffetaut E. 2007. Rediscovery and redescription of the holotype of Aepysaurus elephantinus Gervais 1848-1852 (Dinosauria-Sauropoda). In: J Le Loeuff, ed. 5th Meeting of the European Association of Vertebrate Palaeontologists, 12th European Workshop of Vertebrate Palaeontology, CarcassoneEspéraza, May 15-19, 2007, Abstract volume, 64.

Upchurch P, Barrett PM, Dodson P. 2004. Sauropoda. In : Weishampel DB, Dodson P, Osmolska H, eds. The Dinosauria, second edition. Berkeley: University of California Press, 259-322.

Vadet A, Rose J-M. 1986. Catalogue commenté des types et figurés de dinosauriens, ichthyosauriens, sauroptérygiens, ptérosauriens et chéloniens du Musée d'Histoire naturelle de Boulogne-sur-Mer. Mémoires de la Société Académique du Boulonnais 1(2): 85-97, pl. $18-19$.

Valenciennes A. 1863. D'une espèce de chélonien fossile d'un genre nouveau, trouvé dans la craie du cap de la Hève par M. Lennier, du Havre. Comptes Rendus hebdomadaires des Séances de l'Académie des Sciences 56: 317-322.

Villeneuve-Flayosc $\mathrm{H}$ de. 1856. Description minéralogique et géologique du Var et des autres parties de la Provence avec application de la géologie à l'agriculture, au gisement des sources et des cours d'eau. Paris: Victor d'Almont, (iv), xxviii, 532 p., 2 pl.

Widenmann E. 1837. Geologische Notizen. Das Ausland $305\left(1^{\mathrm{er}}\right.$ novembre 1837): 1220 .

Wiegmann A. 1837. Bericht über die Leistungen im Gebiete der Zoologie während des Jahres 1836. Archiv für Naturgeschichte 2: 125-254.

\section{Annexe 1}

Transcription d'une lettre de Bruno Marmin (BNF NAF 24251/folios 282-283) adressée «à Monsieur Jules Desnoyers, Bibliothécaire du muséum d'histoire naturelle, au Jardin du Roi, à Paris ».

[début de la page 1, folio 282, recto] Boulogne sur Mer Juillet 1835 Monsieur.

Vous êtes venu passer quelques instans [sic] à Boulogne et mon neveu ${ }^{1}$ plus heureux que moi a profité seul de votre méprise. Je prends cependant pour moi votre regret obligeant et je prie de croire que je le partage.

\footnotetext{
${ }^{1}$ Charles Alexandre Marmin (1806-1873), conservateur du Musée de Boulogne-sur-Mer

${ }^{2}$ Son épouse, Anne Joséphine Marmin, née Landes, venait de décéder, trois ans et demi plus tôt, le 5 janvier 1832 à Lille (Nord), à l'âge de 43 ans. Anne Joséphine Landes naquit le 29 novembre 1788 à Dijon (Côte-d'Or). Elle est la fille de Pierre Landes, avocat à la cour, et d'Anne Regneau.

${ }^{3}$ Son frère Alexandre Marmin (1780-1830) mourut à Boulogne-surMer le 10 septembre 1830.
}

J'habite cette ville depuis deux ans, après avoir perdu dans l'espace de six mois, ma femme ${ }^{2}$, mon frère unique ${ }^{3}$, deux amis intimes et mon emploi d'inspecteur des postes, par suite de la suppression de mes 30 collègues.

Je suis arrivé ici dans un état affreux, ayant le dégout de la vie et la certitude d'un prochain repos. On m'a entouré, on m'a rappelé que j'ai un fils, des parins [sic] qui m'aiment, et qq. [quelques] amis dont l'affection est restée invariable: on m'a promené dans nos montagnes, on $m$ 'a forcé à me fatiguer l'esprit et le corps, on m'a donné des pétrifications, ce qui était offrir du vin à un ivrogne, et ma douleur est devenu de la résignation.

Ce récit doit vous faire deviner, Monsieur, que j'ai repris mes études d'H $H^{\text {re }} n^{\text {elle }}$ [Histoire naturelle] et que le plaisir de vous voir et d'en causer avec vous, eut été pour moi un bonheur véritable. Je souhaite que le gout de la science vous ramène parmi nous; vous n'aurez pas à regretter, j'en suis certain, d'être venu visiter nos curieuses localités. J'ai commencé la formation d'une collection qui comprendra tous les débris fossiles de l'arrondissement de Boulogne, et je m'apperçois [sic] déjà qu'elle aura de l'intérêt car le Pays est fort riche en ce genre. Je ne veux pas renoncer à l'étude de la conchyliologie, mais j'ai l'intention d'y ajouter celle de la géologie. La crainte d'avoir encore au bout de ces deux moyens de distraction, une seule minute de tems à donner à ma mélancolie, fait que je m'occupe en outre à former un médailler des monnaies du moyen âge. Vous voyez, Monsieur, combien la complaisance d'autrui me sera préçieuse [sic] pour augmenter ces trois collections distinctes! Je réclame la vôtre avec confiance, parce que j'espère pouvoir de mon côté vous offrir, en échange des vôtres, des objets qui pourront avoir de l'intérêt à vos yeux. J'attends la fin des chaleurs pour continuer mes explorations.

[début de la page 2, folio 282, verso] J'ai trouvé des térébratules d'une conservation parfaite qui sont au nombre de 10 ou 12 espèces différentes. 4 ou 5 espèces $d u g^{r e}$ [genre] Gryphaea dont une bien certainement inédite: sa valve sup ${ }^{\text {re }}$ [supérieure] est comme guillochée. La griphée gondole (cymbium) est d'une épaisseur remarquable. La bélemnite naine est commune ici, mais il en est une autre plus rare que l'on confond avec elle et que je crois nouvelle. Si un envoi de ces diverses choses peut vous être agréable, je m'empresserai de vous envoyer des échantillons des espèces dont j'aurai des doubles. J'ai découvert des Pernes assez curieuses. Voici un mauvais croquis ${ }^{4}$ d'une espèce d'avicule trouvée dans une formation de la $g^{\text {de }}$ [grande] oolite de Marquise à 3 lieues de Boulogne. C'est le seul exemplaire que je possède: j'ignore son nom. Nous avons un terrain identique avec celui de Fohlkstone (Angl ${ }^{r e}$ ) [Folkestone (Angleterre)] c'est à Wissant qui est placé vis-à-vis. $M^{r}$ Eugène Robert ${ }^{5}$, annonce dans le Bulletin de la Société Géologique du 4 mars $1834^{6}$, l'existence dans la craie tufacée et chloritée qui existe entre ce dernier endroit et le Cap Blanc-Nez, de diverses espèces d'ammonites : ce qui est vrai mais il annonce avoir trouvé en même tems [sic] six espèces d'hamites, d'une très belle conservation : chose qui

\footnotetext{
${ }^{4}$ La lettre de Marmin est accompagnée d'un dessin (BNF NAF 24251/ folio 284) représentant une valve d'Oxytoma (Avicula) sp. «de la carrière de Marquise près Boulogne (Pas de Calais)» (Bathonien). ${ }^{5}$ Eugène Robert (1806-1882).

${ }^{6}$ (Robert, 1834 ; Rozet, 1835, p. li)
} 
me parait assez douteuse, car mes recherches à ce sujet ont été maleureuses. Le résultat a été pour moi, qq [quelques] tronçons presqu'indéterminables. L'H. [Hamites] cylindricus seulement s'obtient qqfois [quelquefois] en bon état. J'en possède un exemplaire parfait, mais chose étrange, il ne ressemble nullement aux exempl ${ }^{\text {res }}$ [exemplaires] que je dois à la complaisance de $\mathrm{Mr}$ de Gerville ${ }^{7}$, car la différence d'aspect doit me faire douter que ce soit la même espèce.

Un de mes amis possède ici une collection considérable d'ossemens [sic] fossiles de grands sauriens ${ }^{8}$. De mon côté j'ai découvert des restes de beaucoup d'animaux. Je me suis procuré une grande dent de Megalosaurus parfaitement conservée malgré deux ruptures. Elle est absolument semblable à celle figurée dans Cuvier tom [sic] 5, $2^{\text {ème }}$ part. Page 338 à 345, pl. 21, fig. $13^{9}$. Mais elle est beaucoup plus grande. Sa taille dépasserait en plus la conjecture de mesure de $M^{r}$. Cuvier, sur une proportion de 50pieds donnée à l'animal d'Oxford cité par lui.

Un anglais de ma connaissance croit qu'on ne connait que celui découvert à Oxford ${ }^{10}$ et il prétend que ma dent vaut mieux pour moi qu'une dent d'or. Je doute de la vérité du fait et je vous aurai obligation de vouloir me donner la possibilité de détruire son erreur. Au surplus, voiçi [sic] la dimension de cette dent : [croquis]

[début de la page 3, folio 283, recto] J'en ai trouvé d'autres fort petites que je crois appartenir à des rongeurs. Elles sont très bien conservées : je pourrai vous en envoyer. Je n'ose pas vous proposer, Monsieur, une correspond ${ }^{c e}$ [correspondance] scientifique, je ne serais pas de force: la géologie m'est encore étrangère et vous avez d'autres choses à faire que d'entreprendre l'éducation d'un ignorant. Je me borne à vous offrir les curiosités de ma Province et je me borne à réclamer de vous et de vos amis, $q q$ [quelques] bribes de vos collections. J'ai besoin d'avoir autour de moi des moyens d'occupations, et les productions de la nature sont celles qui ont l'avantage de me séduire. Quelques lignes de tems à autres et vous comblerez tous mes voux. Ces messieurs de Paris me regardent sans doute en pitié ou comme un homme mort ou inutile à leurs yeux. $M^{r}$. Michelin ${ }^{11}{ }^{1}$ 'a planté la [là], $M^{r}$. Félix de Roissy ${ }^{12} a ̀$ qui je pouvais offrir des ammonites n'a pas daigné répondre depuis deux ans à ma dernière lettre : je le laisse en repos. $M^{r}$. Dorbigny ${ }^{13}$ m'avait promis des doubles de ses coquilles

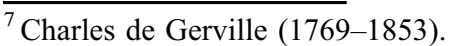

${ }^{8}$ Il pourrait s'agir de la collection de Pierre Nicolas Maurice DutertreDelporte (1788-1862) connue pour avoir été riche en restes de reptiles (crocodiliens, sauroptérygiens et dinosauriens) (Rose et Vadet, 1986; Vadet et Rose, 1986).

${ }^{9}$ (Cuvier, 1824).

${ }^{10}$ Il est fait ici allusion aux restes du Megalosaurus bucklandii Mantell, 1827 découverts à Stonesfield, Oxfordshire, et décrits par William Buckland (1824; Benson et al., 2008; Benson, 2010; Spalding et Sarjeant, 2012).

${ }^{11}$ Jean-Louis Hardouin Michelin de Choisy (1786-1867).

${ }^{12}$ Augustin Félix Pierre Michel de Roissy (1771-1843).

${ }^{13}$ Alcide Charles Victor Marie Dessalines d'Orbigny (1802-1857).

${ }^{14}$ Alcide d'Orbigny revenait d'un voyage scientifique de sept années et sept mois en Amérique du Sud. Il était revenu en France depuis janvier 1834 et se consacrait à la rédaction de son ouvrage Voyage en Amérique méridionale publié entre 1835 et 1847.
}

terrestres et fluviatiles exotiques. Je lui ai écrit à son retour ${ }^{14}$ pour le féliciter et lui demander son fretin. Je ne connais son existence que par la publication de son ouvrage. J'ai l'espoir, Monsieur, que vous, qui étiez si estimé de Mons. Duvau ${ }^{r}$, vous aurez le tems et la volonté de répondre à l'expression de mes sentimens [sic] pour vous et aux sollicita[tions] que je prends la liberté de vous adresser. Vous saurez comprendre qu'[un] motif plus impérieux que celui de qq distractions, m'oblige à m'oc [cuper] l'esprit et que j'ai besoin de quelques affections consolantes pour ne pas perdre le fruit de ma résignation à mes malheurs.

Malgré l'intérêt du muséum de Boulogne et celui d'une Bibli $^{\text {que }}$ [bibliothèque] assez belle, les livres $d^{\prime} h^{\text {re }} n^{\text {elle }}$ [histoire naturelle] sont rares et les amateurs peu nombreux: nous n'avons rien sur la géologie et les fossiles. Voici ce que je souhaite: les coquilles vivantes surtout terrestres et fluviatiles exotiques nouvellement découvertes et devenues assez communes pour pouvoir en obtenir sans être indiscrets. Les ammonites, hamites, nautilites, bélemnites de tous les terrains ainsi que les pétrifications d'Angl' [Angleterre], de Normandie et d'Allemagne, des fossiles d'Italie \&c \&c les plantes fossiles, les impressions de fougères, les poissons pétrifiés, les trilobites et ce qui a trait aux animaux perdus. Je ne voudrais pas d'objets d'un trop gros volume. Si vous aviez ensuite de vieilles monnaies des ducs de Normandie, de Bretagne et autres du moyen âge, à m'adresser contre l'équivalent de vos achats, ce serait me rendre fort heureux. Enfin, Monsieur, si dans vos brochures scientifques, vous en aviez de doubles ainsi que des gravures dépareillées, tout cela serait parfait pour moi.

En attendant le plaisir de recevoir de vos nouvelles, veuillez croire, Monsieur, à ma haute et parfaite considération.

Marmin

Ancien inspecteur des postes

rue d'Aumont $n^{\circ} .4$.

Veuillez S.V.P. présenter mes compliments empressés à $M^{r}$. de Blainville ${ }^{16}$ à qui j'offre aussi des pétrifications.

Si votre muséum était moins complet, je pourrais lui demander qq [quelque] chose de son magasin contre nos curiosités naturelles.

\section{Annexe 2}

La famille Marmin est signalée dans l'actuel département du Pas-de-Calais, à Hardinghen, dès les années 1670 (Lefèbvre, 1908, p. 59). Le grand père de Bruno Marmin, Charles Marmin (mort le 12 mai 1753), commerçant à Boulogne, se maria une première fois le 31 janvier 1719 avec Antoinette Benoîte Gressier (décédée le 17 mars 1732 à l'âge de 45 ans environ) dont il eut cinq enfants, puis le 26 juillet 1740 avec Marie Anne Libert (ADPC, registres 5MIR160/10/1，5MIR160/10/2, 5MIR160/46 et 5MIR160/47). De ce second lit, il eut deux enfants, Lucie Marmin (née le 22 janvier 1742) et Louis Marie François Marmin (2 août 1744-8 février 1826), directeur des postes à Boulogne, qui se maria le 23 novembre 1779 avec Marie Jeanne Pihen (12 juin 1751-29 décembre 1817). De cette union, naquirent Alexandre Marmin (26 août 1780 10 septembre 1830) et Bruno Marmin (20 avril 1782-

\footnotetext{
${ }^{15}$ Auguste Duvau (1771-1831).

${ }^{16}$ Henri Marie Ducrotay de Blainville (1777-1850).
} 
13 septembre 1866) (ADPC, Boulogne sur mer, décès, 18251833, 5 MIR 160/47; 1865-1870, 5 MIR 160/53). L'ainé de la famille, Alexandre, fut membre du conseil municipal de Boulogne-sur-Mer et se fit connaître en son temps par la publication de plusieurs dissertations sur l'archéologie et l'histoire. Son fils (donc le neveu de Bruno Marmin), Charles Alexandre Marmin (21 décembre 1806-28 mai 1873), fut conservateur du Musée de Boulogne-sur-Mer et contribua activement à l'enrichir en objets d'art, antiquités, médailles et monnaies anciennes (Haigneré, 1873).

\section{Annexe 3}

Transcription d'une lettre de Prosper Renaux à Henri Marie Ducrotay de Blainville (BCM Ms BLA 9) adressée «à Monsieur de Blainville, professeur au Muséum d'Histoire naturelle, à Paris ».

[début de la page 1] Avignon le 13 août 1842

Monsieur

Je profite de l'occasion qui m'est offerte par Monsieur Mérimée ${ }^{17}$ inspecteur des monumens historiques pour vous adresser le dessin que j'ai fait de l'ossement que j'ai découvert dans les Sables verts du mont Ventoux au lieu de Bedoin. J'ai fait une petite notice sur cet os qui est vraisemblablement d'un Cétacé ou peut être d'un Saurien et il me serait bien agréable d'avoir votre avis sur ce point afin de le citer dans mon petit opuscule. J'espère Monsieur que vous ne me refuserez pas le concours de votre haute science. Si la détermination de mon os est difficile vous êtes celui parmi tous les savants qui [début de la page 2] pouvez le mieux faire cette détermination.

J'ai fait mouler cet os etj'en ai coloré un fac-simile que je destine au muséum. J'ai préparé également une petite caisse dans laquelle j'ai rangé 35 échantillons d'ossements d'hipparion et autres du terrain lacustre supérieur à la molasse de Cucurron [sic] (Vaucluse) et une quinzaine d'échantillons du terrain à gypse de Gargas qui est inférieur à la plus ancienne molasse et se trouve l'analogue du gypse de Montmartre et d'Orléans.

Vous trouverez ensuite dans le même envoi une caisse contenant des coquilles fossiles pour le muséum et que vous aurez l'obligeance de faire remettre à $M^{r}$ Valenciennes chargé de la section de conchologie [sic]. J'ai taché de lui adresser la série de ce département dont il a déjà reçu une assez grande partie de Requien ${ }^{18}$. Les échantillons du $n^{\circ} 51$ au $n^{\circ} 235$ sont destinés à $M^{r}$ Valenciennes ${ }^{19}$.

Le surplus compris dans la caisse $n^{\circ} 3$ et qui sert de remplissage à la grande caisse est destiné à la collection géologique. Veuillez [début de la page 3] bien en faire la remise $\grave{a}$ Monsieur Cordier ${ }^{20}$. Il y a plus de 6 mois que ces fossiles sont préparés pour vous être adressé mais je ne savais pas de quelle manière je devais faire cet envoi. Veuillez Monsieur m'en écrire et je me cantonnerai à vos ordres.

\footnotetext{
${ }^{17}$ Prosper Mérimée (1803-1870).

${ }^{18}$ Esprit Requien (1788-1851). Dans une lettre datée du 23 janvier 1844 (BCM Ms BLA 9), Requien mentionna également à Blainville la découverte de l'humérus de sauropode du mont Ventoux : « $\mathrm{M}^{\mathrm{r}}$ Renaux notre architecte du dep ${ }^{t}$ a trouvé un énorme ossement de cétacé je crois dont il vous offrirait une épreuve si cela vous était agréable».

${ }^{19}$ Achille Valenciennes (1794-1865).

${ }^{20}$ Louis Cordier (1777-1861).
}

En vous envoyant nos produits nous désirons autant que possible que ce soit en faveur de la science et afin de faciliter les comparaisons qui servent à l'étendre en développant les idées pour les généraliser, aussi j'insisterai auprès de vous, Monsieur, pour obtenir de votre bonté la liste des objets que je vous adresse avec la détermination des noms et celui des auteurs. Vous voudrez bien marquer d'une astérie [sic] les fossiles qu'il vous serait agréable de recevoir pour votre collection particulière ou pour vos amis. Je m'empresserai de vous les adresser.

De mon côté je désire obtenir en échange de ce que je vous envoie, quelques doubles des terrains crétacés du Nord, soit en coquilles, soit en ossemens, ou crustacés, [...] des ossemens de vos terrains tertiaires, enfin j'aimerai bien aussi obtenir des fossiles Jurassique supérieur si cela est possible.

Veuillez, Monsieur, excuser mon indiscrétion de ce que je viens ainsi vous troubler au milieu de vos sérieuses occupations. Il y a 18 ans que je m'occupe de ramasser. Il y en a 12 que j'étudie la géologie dont je suis ardent amateur. À ce titre vous me pardonnerez [début de la page 4] la peine que je vais vous occasionner. En attendant votre réponse, veuillez croire à la haute considération avec laquelle j'ai l'honneur d'être, Monsieur, votre humble et très obéissant serviteur

$P^{\text {er Renaux }}$

( $a r c^{\text {te }}$ du dépt. de Vaucluse, rue Bonneterie $n^{\circ} 27$ à Avignon) Je n'enverrai la caisse que lorsque vous m'aurez fait l'honneur de me répondre.

\section{Annexe 4}

Cette annexe donne la liste chronologique des premiers dinosaures, ou supposés comme tels, trouvés en France avant 1842, année de création du terme Dinosauria par Richard Owen. Le symbole $\left[{ }^{*}\right]$ indique que l'occurrence est attestée par l'existence de figures publiées ou de dessins. Le symbole (§) indique qu'il n'est pas prouvé qu'il s'agisse bien de restes de dinosaures (spécimens non vus ou absence d'illustrations) et que l'occurrence est donc hypothétique. Le symbole ( $\S \S)$ indique qu'il n'existe pas de preuves formelles que les spécimens retrouvés sont bien ceux dont il est question.

en ou avant $1776[\S]$ :

- collecteur: Jacques-François Dicquemare (1733-1789);

- localité et horizon: Vaches Noires (Calvados), Marnes de Dives ou de Villers (Callovien supérieur/Oxfordien inférieur);

- spécimen supposé : hypothétique fémur de dinosaure (non figuré) (Taquet, 1984).

avant 1795 (probablement en ou avant 1778) [*]:

- collecteur: Charles Bacheley (1716-1795);

- localité et horizon: Vaches Noires, Marnes de Dives ou de Villers (Callovien supérieur/Oxfordien inférieur);

- spécimens authentifiés: vertèbres de Streptospondylus altdorfensis figurées par Cuvier (1808) et indiquées comme appartenant à la collection Bacheley: MNHN.F. RJN78, 79, 85, 86 (Allain, 2001 ; Brignon, 2016a, 2016b);

- autres spécimens supposés : autres vertèbres, pubis, tibia, astragale et calcanéum de Streptospondylus altdorfensis répertoriés par Allain (2001) non explicitement indiqués par Cuvier comme faisant partie de la collection Bacheley. 
en ou avant avril $1826\left[{ }^{*}\right]$ :

- collecteur: Arcisse de Caumont (1801-1873);

- localité et horizon: Quilly (Calvados), Calcaire de Caen (Bathonien moyen);

- spécimen authentifié: dent de théropode figurée par de Caumont (1828, pl. 8);

- autres spécimens supposés: autres dents de théropode.

en ou avant octobre $1826[\S \S]$ :

- collecteur: inconnu. Spécimens identifiés par William Buckland (1784-1856) et signalés dans une publication pour la première fois par De la Beche (1830a);

- localité et horizon: Puits de Brème, Scey-Maisières (Doubs), oolithes ferrugineuses (Callovien);

- spécimens supposés : un fragment de «mâchoire » avec des dents et quelques autres os de «Megalosaurus» de l' «oolite» de la région de Besançon (Buckland, 1836, vol. 1, p. 234). Un sacrum et des vertèbres de théropodes (Theropoda indéterminé) conservés dans les collections paléontologiques de l'Université de Besançon pourraient faire partie de ce matériel (Buffetaut et al., 1994 ; Taquet et Contini, 1997).

$1831[\S]$ :

- collecteur: Charles Bertrand-Geslin (1796-1865);

- localité et horizon : Belle-Croix, Dompierre-sur-Mer (Charente-Maritime), calcaires

du Kimméridgien inférieur;

- spécimen supposé : hypothétique vertèbre de «Megalosaurus » (non figurée).

avant juillet $1833[\S]$ :

- collecteur: Frédéric de La Fresnaye (1783-1861);

- localité et horizon: Épaney (Calvados), calcaires du Bathonien;

- spécimen supposé : hypothétique os long de «Megalosaurus » (non figuré).

automne 1833 [§]:

- collecteur: Eugène Robert (1806-1882);

- localité et horizon: Boulogne-sur-Mer, Jurassique supérieur;

- spécimens supposés : hypothétique dent de «Megalosau$r u s »$ et autres spécimens qui pourraient correspondre à une dent de dinosaure herbivore et à divers ossements de dinosaures (non figurés).

en ou avant juillet 1835 [*]:

- collecteur: Bruno Marmin (1782-1866);

- localité et horizon: Fort de Croï, Wimereux, Assises de Croï (Tithonien);

- spécimen authentifié: dent de théropode (Theropoda indéterminé) figurée dans la lettre de Marmin à Desnoyers
(Fig. 2). Cette pièce était conservée au Musée d'Histoire naturelle de Boulogne-sur-Mer (MHNB 122, ancien numéro 309).

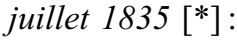

- collecteurs : Alexandre Frédéric Bourrienne (1797-1871) \& Jacques-Amand Eudes-Deslongchamps (1794-1867);

- localité et horizon : carrières de La Maladrerie (Calvados), Calcaire de Caen (Bathonien moyen);

- spécimen authentifié : squelette partiel de Poekilopleuron bucklandii Eudes-Deslongchamps, 1836a figuré par J.-A. Eudes-Deslongchamps, (1837a, 1838). Moulages en plâtres conservés au MNHN et au Yale Peabody Museum of Natural History (Allain et Chure, 2002).

avant janvier $1839[\S]$ :

- collecteur: Henri Coquand (1813-1881);

- localité et horizon: Le Val (Var), formations fluviatiles gréseuses du Bégudien?;

- spécimens supposés: deux hypothétiques os de grandes dimensions que Coquand (1839) identifiait comme des fémurs de mastodonte.

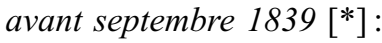

- collecteur: Pierre Nicolas Maurice Dutertre-Delporte (1788-1864)-découverte signalée par Constant Prévost (1787-1856);

- localité et horizon: La Poterie, près de Wimille (Pas-deCalais), Tithonien;

- spécimen authentifié: extrémité d'un os (radius?) de sauropode [Sauropoda indéterminé] figurée par Seeley (1880). Cette pièce était conservée au Musée d'Histoire naturelle de Boulogne-sur-Mer.

en ou avant 1841 [*]:

- collecteur: Simon Bornet;

- localité et horizon: plaine de «Lisle» près de Périgueux (Dordogne), Crétacé supérieur ;

- spécimens authentifiés : fragments d'os longs de sauropode (s) [Sauropoda indéterminé(s)] figurés par Gervais (18481852, vol. 3, pl.63, fig. 1-2).

$1841\left[{ }^{*}\right]:$

- collecteur: Prosper Renaux (1793-1852);

- localité et horizon: Bédoin, Mont-Ventoux (Vaucluse), grès vert (Albien);

- spécimen authentifié : humérus de sauropode (holotype d'Aepisaurus elephantinus) figuré par Renaux (Fig. 4) et Gervais (1848-1852, vol.3, pl.63, fig. 3-4). La partie proximale de cette pièce est conservée à l'Université de Montpellier (BED01) et un moulage en plâtre du spécimen complet au MNHN (1868-242). 